\title{
Functional responses for zooplankton feeding on multiple resources: a review of assumptions and biological dynamics
}

\author{
Wendy Gentleman ${ }^{\mathrm{a}, *}$, Andrew Leising ${ }^{\mathrm{b}}$, Bruce Frost ${ }^{\mathrm{c}}$, Suzanne Strom ${ }^{\mathrm{d}}$, \\ James Murray ${ }^{\mathrm{c}}$ \\ ${ }^{a}$ Engineering Mathematics, Dalhousie University, 1340 Barrington Street, Halifax, NS, Canada B3J 2 X4 \\ ${ }^{\mathrm{b}}$ Pacific Fisheries Environmental Laboratory, 1352 Lighthouse Ave., Pacific Grove, CA 93950, USA \\ ${ }^{\mathrm{c}}$ School of Oceanography, University of Washington, Box 357940, Seattle, WA 98195-7940, USA \\ ${ }^{\mathrm{d}}$ Shannon Point Marine Center, 1900 Shannon Point, Anacortes, WA 98221, USA
}

Received 3 May 2002; received in revised form 2 April 2003; accepted 15 July 2003

\begin{abstract}
Modelers often need to quantify the rates at which zooplankton consume a variety of species, size classes and trophic types. Implicit in the equations used to describe the multiple resource functional response (i.e. how nutritional intake varies with resource densities) are assumptions that are not often stated, let alone tested. This is problematic because models are sensitive to the details of these formulations. Here, we enable modelers to make more informed decisions by providing them with a new framework for considering zooplankton feeding on multiple resources. We define a new classification of multiple resource responses that is based on preference, selection and switching, and we develop a set of mathematical diagnostics that elucidate model assumptions. We use these tools to evaluate the assumptions and biological dynamics inherent in published multiple resource responses. These models are shown to simulate different resource preferences, implied single resource responses, changes in intake with changing resource densities, nutritional benefits of generalism, and nutritional costs of selection. Certain formulations are further shown to exhibit anomalous dynamics such as negative switching and sub-optimal feeding. Such varied responses can have vastly different ecological consequences for both zooplankton and their resources; inappropriate choices may incorrectly quantify biologicallymediated fluxes and predict spurious dynamics. We discuss how our classes and diagnostics can help constrain parameters, interpret behaviors, and identify limitations to a formulation's applicability for both regional (e.g. HighNitrate-Low-Chlorophyll regions comprising large areas of the Pacific) and large-scale applications (e.g. global biogeochemical or climate change models). Strategies for assessing uncertainty and for using the mathematics to guide future experimental investigations are also discussed.
\end{abstract}

(C) 2003 Elsevier Ltd. All rights reserved.

Keywords: Plankton dynamics; Functional response; Zooplankton grazing; Preference; Selection; Switching

\footnotetext{
*Corresponding author. Tel.: + 1-902-494-6086; fax: + 1-902423-1801.

E-mail address: wendy.gentleman@dal.ca (W. Gentleman).
}

\section{Introduction}

Models of planktonic populations and ecosystems traditionally consider zooplankton as feeding upon a single nutritional resource (i.e. only one 
input to a "zooplankton box", Fig. 1a) even though their natural diets are usually comprised of a mixture of trophic types, species, size-classes, and detritus. However, models need to explicitly describe the ingestion of multiple resources (i.e. more than one input, Fig. 1b) in order to assess the importance of omnivory, to estimate secondary production, and to predict plankton dynamics in regions where zooplankton are food-limited. Quantifying both the total nutritional intake and how that intake is derived from the various resources is complicated because many factors contribute to the functional response (i.e. the way intake changes with resource density; Solomon, 1949).
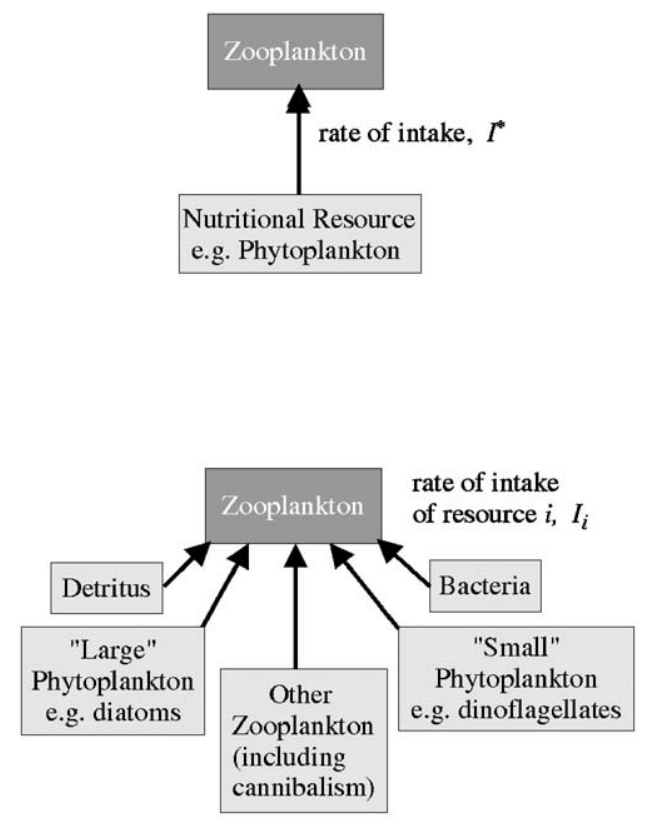

Fig. 1. Schematics of nutritional resources for zooplankton. The number of nutritional resources explicitly considered by a given model can easily be determined through examination of the model's schematic. (a) Models that consider zooplankton feeding on a single resource (e.g. phytoplankton) have only one arrow pointing to a "zooplankton box", and the specific rate of intake is dictated by the single resource functional response, $I^{*}$. (b) Models with more than one arrow pointing to a "zooplankton box" consider zooplankton feeding on multiple resources, such as different trophic types, species, size-classes and/or detritus. In these models, the specific rate of intake of resource $i$ is dictated by the multiple resource responses $I_{i}$, and in this example since there are 5 arrows, $i=1,2, \ldots, 5$.
Zooplankton can exhibit a different functional response for each resource when that resource is the only nutrition available (i.e. different single resource responses) due to differences in the predators' ability to perceive and capture specific prey (Green, 1986; Jonsson and Tiselius, 1990; DeMott and Watson, 1991). Different single resource responses also arise from differences in the resources' nutritional content or time-scales for their handling and assimilation (Fenchel, 1980; Jonsson, 1986). The intake rate for any one resource may additionally be affected by the presence of other resources, such as when the time devoted to one is restricted by the time devoted to others or when behavioral changes occur with variations in relative resource densities (Donaghay and Small, 1979; Ambler, 1986; Colton, 1987; Gifford and Dagg, 1988; Verity, 1991, Kiorboe et al., 1996; Strom and Loukos, 1998). Responses may further be influenced by environmental factors such as temperature and turbulence (Rothschild and Osborn, 1988; Davis et al., 1991; Kiorboe et al., 1996; Campbell et al., 2001; Sell et al., 2001).

The convolution of such factors makes it virtually impossible to determine the multiple resource functional response from field data. Experimental determination requires measurement of the nutritional intake for ranges of combinations of resource densities (Colton, 1987). Unfortunately, few such factorial design experiments have been performed, leaving us with very limited knowledge. As a result, most models of multiple resources are based on explicit assumptions about how single resource responses can be extended (e.g., prescribing additional parameters or density dependencies). However, implicit in the resulting equations are other assumptions that are not often stated, let alone tested. This makes it difficult to choose an appropriate equation and to quantify the uncertainty due to ignorance about the actual response, which is problematic because models are sensitive to the details of these formulations (Jost et al., 1973; Oaten and Murdoch, 1975a, b; Matsuda et al., 1986; Franks et al., 1986; Gismervik and Andersen, 1997; Leising et al., 2003). 
Our objective here is to enable and encourage researchers to make more informed decisions, think critically about their choices, and explore the consequences of alternatives. Beginning with a review of the various Types of single resource responses, we then develop a similar classification for multiple resource responses, and present a set of mathematical diagnostics that elucidate model assumptions. A review of published functional responses for zooplankton feeding on multiple resources is presented, and our tools are used to evaluate the assumptions and biological dynamics inherent in those formulations. We consider the implications of different multiple resource responses and make recommendations for modelers who wish to incorporate such ecological structure into their applications. Strategies for assessing how sensitive model results are to the assumptions, and how knowledge of the mathematical dynamics can direct future experimental investigations, also are discussed.

\section{Types of single resource functional responses}

Single resource functional responses relate the specific rate (i.e. per capita zooplankton per unit time) of nutritional intake, $I^{*}$, to resource density, $N$. These models are based on laboratory experiments wherein predator populations are acclimatized to different resource densities, and on theoretical arguments regarding predator behavior and physiology. Holling $(1959,1962,1965)$ described four "Types" of relationships and alternative types have also been observed. Common responses are shown in Fig. 2 and listed in Table 1 along with sample references to where they have been fit to data. In summary:

Type 1 responses exhibit a linear variation of $I^{*}$ with $N$ according to the constant rate of change $r$ (Fig. 2a). Type 1 responses may be Non-Satiating, but are more typically Rectilinear, such that intake reaches a maximum rate $m$ for resource densities above a critical value $v$ (Table 1$)$.

Type 2 responses exhibit a curved variation of $I^{*}$ with $N$ that is concave downward. They have been described by the Disk equation (Holling, 1959 , 1965), which is based on predator-prey theory and is defined by two parameters: handling time $h$ and successful attack rate $a$ (Fig. 2b, Table 1). The latter is the combined rate of encounter, attack, and capture per capita resource and may depend upon factors such as sensory reception, motility, and turbulence (e.g. Rothschild and Osborn, 1988). The Michaelis-Menten equation (Michaelis and Menten, 1913), also called the Monod equation (Monod, 1942, 1950), which is based on enzyme kinetics theory, is mathematically equivalent to the Disk model but is characterized using two different parameters: maximum rate $m$ and half-saturation constant $k$. The latter is the resource density for which the intake is exactly half its maximum (i.e. when $N=$ $k, I^{*}=m / 2$, Fig. 2b). The equivalence of these formulations means that the Michaelis-Menten parameters can be expressed in terms of the Disk parameters (i.e. $m=1 / h, k=1 / a h$, Table 1). Type 2 responses also have been described by the Ivlev equation (Ivlev, 1955), which represents the probability of feeding at the maximal rate $m$ as exponentially distributed with $N$ according to the parameter $d$. The Ivlev model has a different rate of change than the Disk/Michaelis-Menten model, even when their half-saturation values are identical (i.e. even when $d=(\ln 2 / k)$, Fig. 2b, Table 1$)$. While there is generally no statistical basis for choosing one Type 2 model over another (Mullin et al., 1975), there is observational evidence supporting the theory underlying the Disk formulation (e.g. Verity, 1991 and references therein).

Type 3 responses exhibit a curved variation of $I^{*}$ with $N$ that contains a point of inflection separating the concave downward portion of the curve from the portion that is not. Sigmoidal models describe moderate or "S-shaped" Type 3 response (Fig. 2c). The first Sigmoidal model ("Sigmoidal I" in Table 1) assumes the constant attack rate $a$ of the Type 2 Disk equation now varies linearly with resource density according to the constant $c$ (i.e. Disk's $a$ is replaced by $\hat{a}=c N$ ). The second Sigmoidal model ("Sigmoidal II" in Table 1) assumes intake occurs in $\sigma$ steps $(\sigma>1)$, where each step $s$ is described by Type 2 Michaelis-Menten kinetics with half-saturation constant $k_{s}$ and maximum rate $m$ (Jost et al., 1973). When $\sigma=2$, the second Sigmoidal model is 

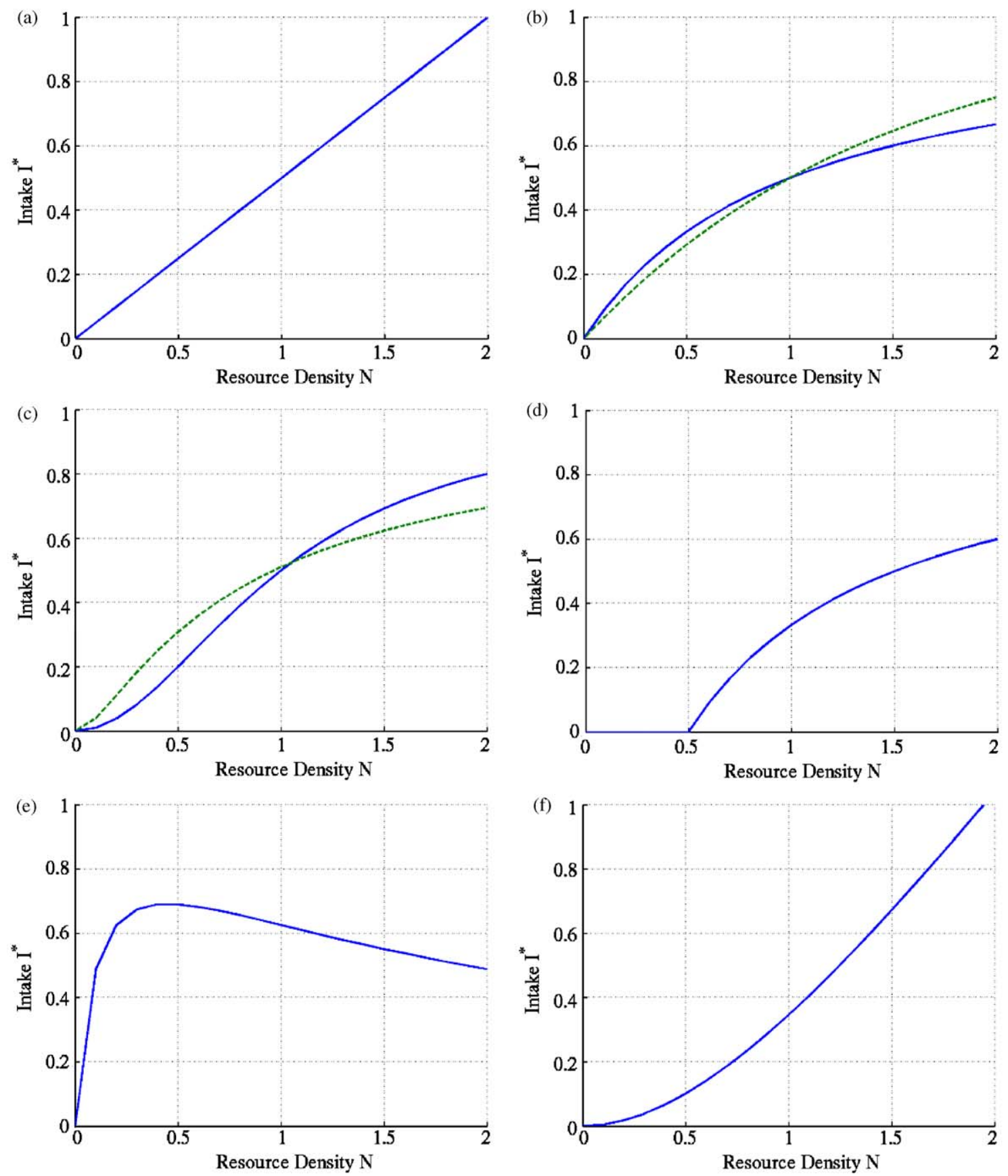

Fig. 2. Single resource functional responses. Plots of $I^{*}$, the nutritional intake associated with a single resource, versus resource density, $N$ (see text and Table 1 for model descriptions). (a) Type 1: Rectilinear $(m=1, v=2)$; (b) Type 2: solid line is Disk/MichaelisMenten $(a=1, h=1$; equivalent to $m=1, k=1)$, dashed line is $\operatorname{Ivlev}(m=1, d=\ln 2)$; (c) Type 3: solid line is Sigmoidal I $(c=1, h=1$; equivalent to $m=1, k=1)$; dashed line is Sigmoidal II ( $\left.m=1, k_{1}=k_{2}=0.4, s=2\right)$; (d) Type 3: Threshold ( $m=1$, $k=1, \tau=0.5)$; (e) Type 4: Toxicity $(\mu=1, \kappa=0.1, \beta=0.25)$; (f) Alternative Type: Modified-Ivlev $(\varepsilon=d=\ln 2)$. 
Table 1

Single resource functional responses.

\begin{tabular}{|c|c|c|c|}
\hline $\begin{array}{l}\text { Single Nutritional } \\
\text { Resource Models }\end{array}$ & Intake & $\begin{array}{l}\text { Parameter } \\
\text { Dimensions }\end{array}$ & $\begin{array}{l}\text { Sample Empirical } \\
\text { References }\end{array}$ \\
\hline (A) Type 1: Non-satiating & $I^{*}=\rho N$ & {$[\rho]=1 /([N] T)$} & $\mathrm{N} / \mathrm{A}$ \\
\hline (B) Type 1: Rectilinear & $I^{*}=\left\{\begin{array}{l}\rho N=\frac{N}{v} m \text { for } N \leqslant v \\
m \text { for } N>v\end{array}\right.$ & $\begin{array}{l}{[\rho]=1 /([N] T)} \\
{[m]=1 / T} \\
{[v]=[N]}\end{array}$ & $\begin{array}{l}\text { Frost (1972), Hansen } \\
\text { and Nielsen (1997), } \\
\text { Mayzaud et al. (1998), } \\
\text { Hansen et al. (1999) }\end{array}$ \\
\hline $\begin{array}{l}\text { (C) Type 2: Disk } \\
\text { a.ka Michaelis-Menten } \\
\text { (a.k.a. Monod) }\end{array}$ & $\begin{array}{l}I^{*}=\frac{a N}{1+a h N} \\
=\frac{N}{k+N} m \\
\text { where } m=1 / h \text { and } k=1 / a h\end{array}$ & $\begin{array}{l}{[a]=1 /([N] T)} \\
{[h]=T} \\
{[m]=1 / T} \\
{[k]=[N]}\end{array}$ & $\begin{array}{l}\text { Mullin et al. (1975), } \\
\text { Ohman (1984), Jonsson (1986), } \\
\text { Mayzaud et al. (1998), } \\
\text { Verity (1991) }\end{array}$ \\
\hline (D) Type 2: Ivlev & $I^{*}=(1-\exp (-d N)) m$ & $\begin{array}{l}{[m]=1 / T} \\
{[d]=1 /[N]}\end{array}$ & $\begin{array}{l}\text { Deason (1980), Barthel (1983), } \\
\text { Houde and Roman (1987) }\end{array}$ \\
\hline (E) Type 3: Threshold & $\begin{array}{l}I^{*}=\frac{N_{e f f}}{k+N_{e f f}} m \\
\text { where } N_{\text {eff }}= \begin{cases}0 & \text { for } N<\tau \\
N-\tau & \text { for } N \geqslant \tau\end{cases} \end{array}$ & $\begin{array}{l}{[m]=1 / T} \\
{[k]=[N]} \\
{[\tau]=[N]}\end{array}$ & $\begin{array}{l}\text { Mullin et al. (1975), } \\
\text { Reeve (1977), } \\
\text { Goldman et al. (1989), } \\
\text { Strom (1991), Lessard and } \\
\text { Murrell (1998) }\end{array}$ \\
\hline $\begin{array}{l}\text { (F) Type 3: Sigmoidal I } \\
\text { (from Disk) } \\
\text { (from Michaelis-Menten) }\end{array}$ & $\begin{array}{l}I^{*}=\frac{\hat{a} N}{1+\hat{a} h N}, \text { where } \hat{a}=c N, \\
=\frac{N^{2}}{k^{2}+N^{2}} m, \\
\text { where } m=1 / h \text { and }=\sqrt{N / \hat{a} h}=1 / \sqrt{c h}\end{array}$ & $\begin{array}{l}{[h]=T} \\
{[c]=1 /\left([N]^{2} T\right)} \\
{[m]=1 / T} \\
{[k]=[N]}\end{array}$ & $\begin{array}{l}\text { Frost (1975), Ohman (1984), } \\
\text { Wickham (1995), Gismervik and } \\
\text { Andersen (1997) }\end{array}$ \\
\hline (G) Type 3: Sigmoidal II & $\begin{array}{l}I^{*}=\frac{N^{\sigma}}{\prod_{s=1}^{\sigma}\left(k_{s}+N\right)} m \text {, where } \sigma>1 \\
\text { When } \sigma=2: \\
I^{*}=\frac{N^{2}}{\left(k_{1}+N\right)\left(k_{2}+N\right)} m=\frac{N^{2}}{k^{2}+N^{2}+\alpha N} m \\
\text { where } k=\sqrt{k_{1} k_{2}} \text { and } \alpha=k_{1}+k_{2}\end{array}$ & $\begin{array}{l}{[\sigma]=\mathrm{ND}} \\
{[m]=1 / T} \\
{\left[k_{s}\right]=[N]} \\
{[k]=[N]} \\
{[\alpha]=[N]}\end{array}$ & $\begin{array}{l}\text { (Theoretical Reference) } \\
\text { Jost et al. (1973) }\end{array}$ \\
\hline $\begin{array}{l}\text { (H) Type 4: Prey Toxicity or } \\
\text { Predator Confusion }\end{array}$ & $I^{*}=\frac{N}{\kappa+N+\beta N^{2}} \mu$ & $\begin{array}{l}{[\mu]=1 / T} \\
{[\kappa]=[N]} \\
{[\beta]=1 /[N]}\end{array}$ & $\begin{array}{l}\text { (Bacteria References) } \\
\text { Veldkamp and Jannasch (1972), } \\
\text { Van Gemerden (1974) }\end{array}$ \\
\hline $\begin{array}{l}\text { (I) Alternate Types: } \\
\text { Modified-Ivlev }\end{array}$ & $I^{*}=\left(1-e^{-d N}\right) \hat{m}, \quad$ where $\hat{m}=\varepsilon N$ & $\begin{array}{l}{[d]=1 /[N]} \\
{[\varepsilon]=1 /(T[N])}\end{array}$ & Mayzaud and Poulet (1978) \\
\hline
\end{tabular}

similar to the first, but with an extra term $(\alpha N)$ in the denominator that results in a different rate of change (Fig. 2c). An extreme Type 3 response is described by the Threshold model (Fig. 2d, Table 1), where no intake occurs for resource densities below a feeding threshold $\tau$. This threshold may be biologically justified or may be a proxy for other processes (Strom et al., 2000). For $N>\tau$, the Threshold equation is identical to a MichaelisMenten equation expressed in terms of an effective resource density $N_{\text {eff }}=N-\tau$. Thus, the Threshold model is a Michaelis-Menten response that is shifted to the right such that $N=k+\tau$ when $I^{*}=$ $m / 2$, which makes it inappropriate to refer to the Threshold model's $k$ as the half-saturation constant.

Type 4 responses are the only ones that do not increase monotonically with increasing resource density. Instead, $I^{*}$ reaches a maximum rate $m$ at an intermediate density $N_{\max }$, and decreases for 
higher $N$ (Fig. 2e). The decrease may occur because of resource toxicity or predator confusion, and/or may result from use of higher resource densities in vitro than predators would encounter in situ (i.e. higher than those for which predators have adapted or evolved). Type 4 responses have been described by an equation similar to the Type 2 Michaelis-Menten model, but with additional term in the denominator $\left(\beta N^{2}\right)$ that results in $m$ and the half-saturation value depending on complicated functions of the model parameters $\mu, \kappa$, and $\beta$ (Table 1).

Alternative types include a response that is similar to a Type 3 Sigmoidal model at low $N$, but that never exhibits satiation (Fig. 2f). This has been described by the Modified-Ivlev model (Mayzaud and Poulet, 1978), in which the Ivlev's constant $m$ is replaced by $\hat{m}=\varepsilon N$ (Table 1 ). Since this formulation has no maximum rate, there is no relationship between the Modified-Ivlev $d$ and the half-saturation value of other models.

\section{Classification of multiple resource responses}

The literature discusses multiple resource responses using terms such as preference, switching, passive and active selection, optimal feeding, and generalism. Here we review the definitions of such concepts, and develops a new classification of multiple resource responses that is akin to the various Types of single source responses.

When predators consume $n$ different kinds of resources, the total intake of a particular nutrient (e.g. nitrogen) depends on the nutritional intake derived from each resource. We denote $I_{i}$ as the specific rate (i.e. per capita zooplankton per unit time) of nutritional intake associated with resource $i$, and consider all resource densities, $N_{i}(i=$ $1,2, \ldots, n)$, to be expressed in a common currency (e.g., nitrogen content). Therefore, $I_{\text {tot }}$, the specific rate of total nutritional intake from multiple resources, is defined by

$I_{\mathrm{tot}}=\sum_{i=1}^{n} I_{i}$,

where $I_{i}$ depends on $N_{i}$ and may additionally depend on the density of other resources, $N_{j}(j \neq i)$.
Thus, the way $I_{\text {tot }}$ changes with the density of any one resource depends on the net effect of the associated changes in every resource's functional response.

Many different definitions have been used for preference (e.g. Chesson, 1983, and references therein; Strom and Loukos, 1998). Here we follow Chesson $(1978,1983)$, where the relative contribution of resource $i$ to the total nutritional intake is equated to the relative contribution of $N_{i}$ to a weighted measure of total resource density,

$\frac{I_{i}}{I_{\mathrm{tot}}}=\frac{\phi_{i} N_{i}}{\sum_{r=1}^{n} \phi_{r} N_{r}}$,

where the non-dimensional weights $\phi_{i}$ are defined as the preferences. The composition of the diet, therefore, can be thought of as a random sampling from preferentially-biased resource densities $\phi_{i} N_{i}$.

Preferences are typically normalized such that any one $\phi_{i}<1$, and $\Sigma \phi_{i}=1$. As Chesson (1983) observed, when timescales considered are small enough that resource densities are essentially constant, the normalized preference for resource $i$ can be estimated by

$\phi_{i}=\frac{I_{i} / N_{i}}{\sum_{r=1}^{n} I_{r} / N_{r}}$.

Recognizing that $F_{i}$, the clearance rate of resource $i$, equals $I_{i} / N_{i}$ (Frost, 1972), one can define $\phi_{i}$ in terms of the relative contribution of $F_{i}$ to the total of all $n$ resources' clearance rates, i.e.

$\phi_{i}=\frac{F_{i}}{\sum_{r=1}^{n} F_{r}}$.

It follows that the relative preference for resource $i$ over resource $j$ is

$\frac{\phi_{i}}{\phi_{j}}=\frac{F_{i}}{F_{j}} \quad(j \neq i)$

which is equivalent to the relative contribution those two resources makes to the diet as compared to their relative densities in the environment. Preferential intake of resource $i$ over resource $j$ occurs when $F_{i} / F_{j}>1$, whereas the converse is true when $F_{i} / F_{j}<1$. 
The relative preference of any two resources may be constant or density-dependent because $\phi_{i}$ are constant or density-dependent. The term switching describes scenarios where $F_{i} / F_{j}$ increases with an increase in relative resource density $N_{i} / N_{j}$ (Murdoch, 1969); negative switching occurs when $F_{i} / F_{j}$ decreases when $N_{i} / N_{j}$ increases (Chesson, 1983; Hutson, 1984). Switching means intake rates change disproportionately with changes in resource densities in a way that can have a stabilizing influence on ecological stability (i.e. how robust the ecosystem is to environmental perturbations), and can promote biodiversity through predation refuges for low-density resources (Oaten and Murdoch, 1975a, b; May, 1977; Holt, 1983). In contrast, negative switching can have a destabilizing influence and can lead to resource extinction.

The term selection refers to mechanisms causing predators to choose among available resources. Passive selection relates to factors such as differential resource vulnerability (including prey motility and size), predator perceptual biases, nutritional or toxic content of the resources, and time-scales for resource handling and assimilation (Strom and Loukos, 1998, and references therein). Thus, passive selection among multiple resources arises from factors causing different single resource responses. In contrast, active selection relates to behaviors that depend on the relative densities of multiple resources, such as alternating between ambush and suspension feeding, rejecting less abundant prey, or concentrating search activity on high-density patches (Landry, 1981; Holt, 1983; Strom and Loukos, 1998, and references therein). Passive and active selection are commonly distinguished by the no-switching versus switching nature of the response (Chesson, 1983; Strom and Loukos, 1998). However, this is not a good metric for making this distinction because passive selection may be density-dependent (Landry, 1981; Holt, 1983), and theoretically active selection could result in constant preferences if the behavioral density-dependence canceled in Eq. (5).

Based on the discussion above, we now define three classes of multiple resource responses:

Class 1 (No switching): Responses for which the relative preference of any two resources are density-independent (constant) and therefore no switching occurs.

Class 2 (Passive switching): Responses for which switching arises from passive selection due to density-dependent behaviors associated with the single resource responses.

Class 3 (Active switching): Responses for which switching arises from active selection due to behaviors that depend on the relative densities of two resources in a manner that may not be predicted from knowledge of the single resource responses.

Classification of a multiple resource response depends on factors affecting feeding behavior, which includes total nutritional intake $I_{\text {tot }}$. Most foraging theories assume predators behaviorally adapt in ways that maximize their nutritional gain, as this enhances their ability to compete and would be favored evolutionarily (e.g., Stephens and Krebs, 1986). One way nutritional intake can be maximized is for $I_{\text {tot }}$ to increase whenever resource densities increase. Following Holt (1983), we define optimal feeding as responses which exhibit such a positive dynamic and sub-optimal responses as those for which $I_{\text {tot }}$ decreases when available nutrition increases. Foraging theory argues that there is a selective advantage to generalism (i.e. consuming $n_{g}$ different resources) over specialism (i.e. consuming only a subset $n_{s}<n_{g}$ ) when intake of a wider variety of resources increases $I_{\text {tot }}$ (Holt, 1983). Similarly, preferential selection of highquality resources is advantageous when their improved nutritional content outweighs any cost of selection, such as that due to time lost distinguishing among resources.

\section{Diagnostics for determining the assumed biological dynamics}

We have developed seven simple diagnostics that can assess the biological dynamics inherent in modeled multiple resource functional responses.

\subsection{Diagnostic I: Effective preference}

A model's assumed preferences are diagnosed by dividing each equation for $I_{i}$ by $N_{i}$ to solve for the assumed clearance rates $F_{i}$ and substituting these 
into Eq. (4). Because the influence of any term appearing in all modeled $F_{i}$ is canceled in this equation, modeled preferences often can be assessed using terms that are mathematically simpler than $F_{i}$. For example, the preference formula reduces to a relative measure of attack rates for certain responses (Chesson, 1983). We define effective preference $E_{i}$ as the simplest quantity that can be used in place of $F_{i}$ in Eq. (4) to yield the preference $\phi_{i}$. That is

$\phi_{i}=\frac{E_{i}}{\sum_{r=1}^{n} E_{r}}$,

where $E_{i}$ may equal $F_{i}$ or may be something that is mathematically simpler (e.g., attack rates). It follows that relative preference $\phi_{i} / \phi_{j}=$ $E_{i} / E_{j}(j \neq i)$. Therefore, a multiple resource model assumes no switching occurs between resource $i$ and $j$ when $E_{i} / E_{j}$ is constant (i.e. Class 1 ), whereas switching is assumed when the ratio depends on the density of at least one of the two resources. Switching is assumed to be active (i.e. Class 3) when $E_{i}$ depends on $N_{j}(j \neq i)$, whereas switching may be passive (i.e. Class 2 ) or active when $E_{i}$ only depends on $N_{i}$; determination requires investigation of Diagnostic II.

\subsection{Diagnostic II: Implied single resource response}

The implied single resource response, $I_{i}^{* \mathrm{imp}}$, is the functional response assumed by a multiple resource model when resource $i$ is the only available nutrition. $I_{i}^{* \text { imp }}$ is diagnosed by examining the modeled intake when all other resource densities are zero, i.e.

$I_{i}^{* \mathrm{imp}}=I_{\mathrm{tot}}\left(N_{j}=0\right)=I_{i}\left(N_{j}=0\right), \quad j \neq i$.

All parameters of $I_{i}^{* i m p}$ are prescribed by the actual single resource response, $I_{i}^{*}$, provided the assumed type is correct. Any parameters of $I_{i}$ that do not appear in $I_{i}^{* \text { imp }}$ cannot be predicted from the single resource responses (i.e. active selection), and multiple resource experiments are required to determine parameter values. Active selection also is assumed when behaviors are inconsistent between the single and multiple resource responses, as when attack rates in $I_{i}^{* \text { imp }}$ are constant but those of $I_{i}$ are density-dependent. When both parameters and behaviors are consistent, passive selection is assumed, and preferences can be predicted from single resource responses.

\subsection{Diagnostic III: Change in intake of one resource as its density increases}

A model's assumed rate of change of intake of resource $i$ for small increases in its density is diagnosed by examining the partial derivative

$\partial I_{i} / \partial N_{i}$.

Eq. (8) is equivalent to the slope of the contours of $I_{i}$ versus $N_{i}$ when all other resource densities, $N_{j}(j \neq i)$, are invariant. The intake of resource $i$ always increases with increasing $N_{i}$ when the slope is always positive. Where the slope is negative, a Type 4 kind of toxicity or confusion response is assumed for resource $i$. Where the slope is zero, the density of resource $i$ is assumed to have no effect on its intake.

\subsection{Diagnostic IV: Change in intake of one resource as the density of another increases}

A model's assumed rate of change of intake of resource $i$ for small increases in the density of another resource is diagnosed by examining the partial derivative

$\partial I_{i} / \partial N_{j}, \quad j \neq i$.

Eq. (9) is equivalent to the slope of the contours of $I_{i}$ versus $N_{j}(j \neq i)$ when the densities of all other resources - including $N_{i}$-are invariant. Where the slope is zero, the density of resource $j$ has no effect on $I_{i}$. Where the slope is negative, resource $j$ is assumed to interfere with the intake of resource $i$, as when time spent feeding on $j$ reduces time devoted to $i$. Where the slope is positive, a synergistic effect is assumed, as when behavior or energy gain associated with $j$ increases the ability to detect or capture $i$.

\subsection{Diagnostic V: Change in total nutritional intake as resource density increases}

A model's assumed rate of change of total nutritional intake for small increases in the density 
of a resource $i$ is diagnosed by examining the partial derivative

$\partial I_{\mathrm{tot}} / \partial N_{i}$.

Eq. (10) is equivalent to the slope of the contours of $I_{\text {tot }}$ versus $N_{i}$ when the densities of all other resources $N_{j}(j \neq i)$ are invariant. Total nutritional intake is independent of $N_{i}$ where the slope is zero. Where the slope is positive, feeding is assumed to be optimal. Where the slope is negative, such that total nutritional intake decreases when available nutrition increases, sub-optimal feeding is assumed.

\subsection{Diagnostic VI: Nutritional benefit (or cost) of generalism}

A model's assumptions about the nutritional benefits of generalism, $B_{\text {gen }}$ versus specialism is diagnosed by calculating the difference in the modeled total nutritional intake for the two cases, i.e.

$B_{\mathrm{gen}}=\sum_{g=1}^{n_{g}} I_{g}-\sum_{s=1}^{n_{s}} I_{s}, \quad n_{g}>n_{s}$.

When specialists consume only one resource, $n_{s}=1$, and the second term on the right-hand side of Eq. (11) is equivalent to $I_{i}^{* i m p}$ (Diagnostic II). Where $B_{\text {gen }}$ is positive, generalism is assumed to be nutritionally advantageous, whereas specialism is the better strategy where $B_{\text {gen }}$ is negative. Where $B_{\text {gen }}$ is zero, the assumption is that nutritional costs and benefits are balanced.

\subsection{Diagnostic VII: Nutritional cost (or benefits) of selection}

Resources that elicit identical $I_{i}^{* \text { imp }}$ (Diagnostic II), with respect to both Type and parameters are functionally equivalent. A model's assumptions about the nutritional cost (or benefit) of selecting among such equal quality resources, $C_{\text {sel }}$, is diagnosed by differencing the modeled nutritional intake when multiple resources are perceived as a single nutrient pool versus when they are perceived as distinct, i.e.

$$
\begin{gathered}
C_{\text {sel }}=I^{* \text { imp }}\left(N_{\text {tot }}\right)-I_{\text {tot }}, \\
\text { where } N_{\text {tot }}=\sum_{r=1}^{n} N_{r} .
\end{gathered}
$$

Where $C_{\text {sel }}$ is positive, predators that do not distinguish among functionally equivalent resources are assumed to be more successful. Where $C_{\text {sel }}$ is negative, a model assumes a nutritional benefit to selection. Where $C_{\text {sel }}$ equals zero, there is neither nutritional advantage nor disadvantage to selection.

\section{Published multiple resource functional responses and their assumed dynamics}

Here, we review functional responses for zooplankton feeding on multiple resources that have been used in the literature, and use the diagnostics presented in Section 4 to elucidate their assumed biological dynamics. Examples from each of the three Classes outlined in Section 3 are considered.

\subsection{Examples of Class 1: No Switching models}

Examples of Class 1 models and their associated references are listed in Table 2a. The multiple resource Disk model (Table 2a) is derived by extending the single resource Disk model (Table 1) assuming: (i) predators attack and handle only one resource at a time, and (ii) density-independence of resource-dependent handling times $h_{i}$ and successful attack rates $a_{i}$ (Murdoch, 1973; Bartram, 1980). The multiple resource Disk and Michaelis-Menten models are equivalent formulations expressed in terms of different parameters (Table 2a), as was true for their single resource analogs (Table 1). Unlike the single resource models, however, these multiple resource equations require specification of different numbers of parameters: $2 n\left(a_{i}\right.$ and $\left.h_{i}, i=1, \ldots, n\right)$ for Disk versus $2 n+1\left(m_{i}, p_{i}\right.$ and $\left.k\right)$ for Michaelis-Menten. The extra degree of freedom in the MichaelisMenten model is made clear by dividing its numerator and denominator by $k$, which results in the identical functional response again defined by only $2 n$ parameters (i.e. $P_{i}$ and $m_{i}$, Table $2 a$ ). 
Table $2 \mathrm{a}$

Class 1 multiple resource functional responses

\begin{tabular}{|c|c|c|c|}
\hline Class I Formulation & Intake of resource $i$ & $\begin{array}{l}\text { Parameter } \\
\text { Dimensions }\end{array}$ & Sample References \\
\hline $\begin{array}{l}\text { (A) Disk } \\
\text { a.k.a. Michaelis-Menten }\end{array}$ & $\begin{array}{l}\begin{aligned} I_{i} & =\frac{a_{i} N_{i}}{1+\sum_{r=1}^{n} a_{r} h_{r} N_{r}} \\
& =\frac{p_{i} N_{i}}{k+R} m_{i} \quad \text { where }=\sum_{r=1}^{n} p_{r} N_{r} \\
& =\frac{P_{i} N_{i}}{1+\hat{R}} m_{i} \quad \text { where } \hat{R}=\sum_{r=1}^{n} P_{r} N_{r}\end{aligned} \\
\text { where } m_{i}=1 / h_{i}, \quad P_{i}=p_{i} / k=a_{i} h_{i}\end{array}$ & $\begin{array}{l}{\left[a_{i}\right]=1 /([N] T)} \\
{\left[h_{i}\right]=T} \\
{\left[m_{i}\right]=1 / T} \\
{[k]=[N]} \\
{\left[p_{i}\right]=\text { N.D. }} \\
{\left[P_{i}\right]=1 /[N]}\end{array}$ & $\begin{array}{l}\text { Murdoch (1973), } \\
\text { Frost (1987), } \\
\text { Moloney and } \\
\text { Field (1991), } \\
\text { Verity (1991), } \\
\text { Gismervik and } \\
\text { Andersen (1997), } \\
\text { Strom and } \\
\text { Loukos (1998) }\end{array}$ \\
\hline (B) Threshold & $\begin{array}{l}I_{i}=\left\{\begin{array}{l}\left(\frac{R-\tau}{k+R-\tau}\right) \frac{p_{i} N_{i}}{R} m, \text { for } R>\tau \\
0, \text { for } R<\tau,\end{array}\right. \\
\quad=\left\{\begin{array}{l}\left(\frac{\hat{R}-\hat{\tau}}{1+\hat{R}-\hat{\tau}}\right) \frac{P_{i} N_{i}}{\hat{R}} m, \text { for } \hat{R}>\hat{\tau} \\
0, \text { for } \hat{R}<\hat{\tau}\end{array}\right. \\
\text { where } P_{i}=p_{i} / k \text { and } \hat{\tau}=\tau / k\end{array}$ & $\begin{array}{l}{[m]=1 / T} \\
{[k]=[N]} \\
{\left[p_{i}\right]=\mathrm{N} . \mathrm{D}} \\
{\left[P_{i}\right]=1 /[N]} \\
{[\tau]=[N]}\end{array}$ & $\begin{array}{l}\text { Evans (1988), } \\
\text { Lancelot et al. (2000) }\end{array}$ \\
\hline (C) Ivlev & $\begin{array}{l}\begin{array}{l}I_{i}= \\
\qquad[1-\exp (-d R)] \frac{p_{i} N_{i}}{R} m \text {, where } R=\sum_{r=1}^{n} p_{r} N_{r} \\
\quad=[1-\exp (-\hat{R})] \frac{P_{i} N_{i}}{\hat{R}} m \text {, where } \hat{R}=\sum_{r=1}^{n} P_{r} N_{r}\end{array} \\
\text { where } P_{i}=d p_{i}\end{array}$ & $\begin{array}{l}{[m]=1 / T} \\
{[k]=[N]} \\
{\left[p_{i}\right]=\mathrm{N} . \mathrm{D}} \\
{\left[P_{i}\right]=1 /[N]} \\
{[d]=1 /[N]}\end{array}$ & $\begin{array}{l}\text { Hofmann and } \\
\text { Ambler (1988) }\end{array}$ \\
\hline (D) Rectilinear & 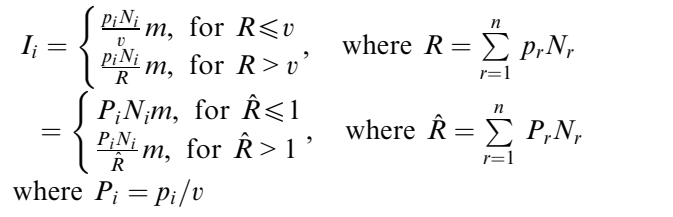 & $\begin{array}{l}{[m]=1 / T} \\
{[v]=[N]} \\
{\left[p_{i}\right]=\text { N.D. }} \\
{\left[P_{i}\right]=1 /[N]}\end{array}$ & Armstrong (1994) \\
\hline
\end{tabular}

\footnotetext{
${ }^{\mathrm{a}}$ Moloney and Field (1991) is included as Class 1: Michaelis-Menten because their model implementation used a single value of the half-saturation constant, $k$, for all resources. However, their generalized equation (their Eq. 3), which allows different half-saturation constants for different resources (i.e. $k_{i}$ ) is actually Class 3: Modified-Michaelis-Menten.
}

The usual justification for such overparameterization is that parameters controlling the dynamics are defined by ones that are easier to measure or comprehend. Nonetheless, overparameterization hides the real influence parameters have on the modeled dynamics.

The Michaelis-Menten equation is one of the most commonly used formulations for zooplankton feeding on multiple resources, and all applications of this model that we cite assume maximum rates are equal for all resources (i.e. all $m_{i}=m$ ). With this restriction, the Michaelis-Menten $I_{\text {tot }}$ takes the form of the single resource MichaelisMenten model (Table 1) expressed in terms of a weighted measure of the total resource density, $R$, i.e.

$I_{\text {tot }}=\frac{R}{k+R} m \quad$ where $R=\sum_{r=1}^{n} p_{r} N_{r}$,

where $p_{r}$ are the weights. In this case, $k$ becomes the value of $R$ when $I_{\text {tot }}=m / 2$, which is why $k$ is called the half-saturation constant in the literature (Fasham et al., 1990; Moloney and Field, 1991; Strom and Loukos, 1998; Loukos et al., 1997; Pitchford and Brindley, 1999). The equal $m_{i}$ restriction allows the Michaelis-Menten $I_{i}$ (Table 2a) to be viewed as the fraction of $I_{\text {tot }}$ that corresponds to the relative contribution of $N_{i}$ to $R$, i.e.

$I_{i}=I_{\mathrm{tot}} \frac{p_{i} N_{i}}{R}$. 
Table $2 b$

Diagnostics of Class 1 examples.

\begin{tabular}{|c|c|c|c|c|c|c|c|}
\hline & $\begin{array}{l}\mathrm{I} \\
E_{i}\end{array}$ & $\begin{array}{l}\text { II } \\
I_{i}^{* i m p}\end{array}$ & $\begin{array}{l}\text { III } \\
\partial I_{i} / \partial N_{i}\end{array}$ & $\begin{array}{l}\text { IV } \\
\partial I_{i} / \partial N_{j}\end{array}$ & $\begin{array}{l}\mathrm{V} \\
\partial I_{\text {tot }} / \partial N_{i}\end{array}$ & $\begin{array}{l}\text { VI } \\
B_{\text {gen }}\end{array}$ & $\begin{array}{l}\text { VII } \\
C_{\text {sel }}\end{array}$ \\
\hline (A) Disk & $\begin{array}{l}a_{i} \\
p_{i} m_{i}\left(\text { or } P_{i} m_{i}\right)\end{array}$ & $\frac{a_{i} N_{i}}{1+a_{i} h_{i} N_{i}}$ & $>0$ always & $<0$ always & $\begin{array}{l}\text { equal } h_{i} \text { or } \\
\text { equal } m_{i}: \\
>0 \text { always }\end{array}$ & $\begin{array}{l}\text { equal } h_{i} \text { or } \\
\text { equal } m_{i}: \\
\geqslant 0 h_{i}>h_{j} \text { or }\end{array}$ & $=0$ always \\
\hline $\begin{array}{l}\text { Michaelis- } \\
\text { Menten }\end{array}$ & & $\begin{array}{l}\frac{N_{i}}{k_{i}^{*}+N_{i}} m_{i} \\
\text { where } k_{i}^{*}=\frac{k}{p_{i}}=\frac{1}{P_{i}}\end{array}$ & & & $\begin{array}{l}h_{i}>h_{j} \text { or } \\
m_{i}>m_{j}:<0 \\
\text { at high } N_{j}\end{array}$ & $\begin{array}{l}m_{i}<m_{j}:<0 \\
\text { at high } N_{j} \text { for } \\
\text { resource } j\end{array}$ & \\
\hline (B) Threshhold & $p_{i}\left(\right.$ or $\left.P_{i}\right)$ & $\begin{array}{l}\text { for } N_{i} \geqslant \tau_{i}^{*}: \\
\qquad \frac{\left(N_{i}-\tau_{i}^{*}\right)}{k_{i}^{*}+N_{i}-\tau_{i}^{*}} m \\
\quad \text { where } k_{i}^{*}=\frac{k}{p_{i}}=\frac{1}{P_{i}} \\
\quad \text { and } \tau_{i}^{*}=\frac{\tau}{p_{i}}=\frac{\tau}{P_{i}} \\
\text { for } N_{i}<\tau_{i}^{*}: 0\end{array}$ & $>0$ always & $\begin{array}{l}\text { near } \tau>0 \\
\text { elsewhere: }<0\end{array}$ & $\geqslant 0$ always & $\geqslant 0$ always & $=0$ always \\
\hline (C) Ivlev & $p_{i}\left(\right.$ or $\left.P_{i}\right)$ & $\begin{array}{l}{\left[1-\exp \left(-d_{i}^{*} N_{i}\right)\right] m} \\
\text { where } d_{i}^{*}=d p_{i}=P_{i}\end{array}$ & $>0$ always & $<0$ always & $\geqslant 0$ always & $\geqslant 0$ always & $=0$ always \\
\hline (D) Rectilinear & $p_{i}\left(\right.$ or $\left.P_{i}\right)$ & $\begin{array}{l}\text { for } N_{i} \leqslant v_{i}^{*}: \frac{N_{i}}{v_{i}^{*}} m \\
\text { for } N_{i}>v_{i}^{*}: m \\
\text { where } v_{i}^{*}=\frac{v}{p_{i}}=\frac{1}{P_{i}}\end{array}$ & $\begin{array}{l}\geqslant 0 \\
\text { always }\end{array}$ & $\begin{array}{l}\text { until } \\
\text { satiated: }=0 \\
\text { once } \\
\text { satiated: }<0\end{array}$ & $\geqslant 0$ always & $\geqslant 0$ always & $=0$ always \\
\hline
\end{tabular}

The multiple resource Threshold, Ivlev, and Rectilinear models (Table 2a), which always assume maximum rates are identical for all resources, are derived in an analogous manner to the Michaelis-Menten models making the same assumption. That is: (i) $I_{\text {tot }}$ is described by each model's respective single resource response (from Table 1) expressed in terms of a weighted measure of total resource density $R$ and (ii) $I_{i}$ is defined by Eq. (14). These three models also are overparameterized, in that the same functional response can be described using one less parameter (i.e. Threshold: $P_{i}=p_{i} / k$, Ivlev: $P_{i}=d p_{i}$, Rectilinear, $P_{i}=p_{i} / v$, Table 2a).

\subsection{Dynamics assumed in Class 1: No Switching examples}

\subsubsection{Diagnostic I}

No Class 1 example ever assumes switching since all their $E_{i}$ are constant, which is why they are in this class (Table 2b). The Disk's $E_{i}$ are the attack rates $a_{i}$ and the Michaelis-Menten's $E_{i}\left(=m_{i} p_{i}\right)$ are the equivalent term scaled by the parameter $k$. Only when the maximum rates $m_{i}$ are identical for all resources do the MichaelisMenten $E_{i}$ simplify to its $p_{i}$ parameters. Thus, despite $p_{i}$ being referred to as "preferences" in the literature (Fasham et al., 1990; Strom and Loukos, 1998; Loukos et al., 1997; Pitchford and Brindley, 1999), the term is a misnomer when any $m_{i}$ are different. Had the Threshold, Ivlev, and Rectilinear models allowed for resource-dependent maximum rates, their $E_{i}$ would also equal $m_{i} p_{i}$, meaning reference to their $p_{i}$ as "selectivities" and "vulnerabilities" (Hofmann and Ambler, 1988; Armstrong, 1994) is somewhat misleading. Furthermore, measured clearance rates will only yield independent estimates of $p_{i}$ in the specific case when all maximum rates are equal.

\subsubsection{Diagnostic $I I$}

All the Class 1 examples assume every resource elicits the same Type of single resource response (e.g. all Disk $I_{i}^{* \text { imp }}$ are Type 2 Disk, all Threshold $I_{i}^{* \text { imp }}$ are Type 3 Threshold, etc., Table $2 \mathrm{~b}$ ). They also all assume selection is passive, as parameters and behaviors are consistent between the single 

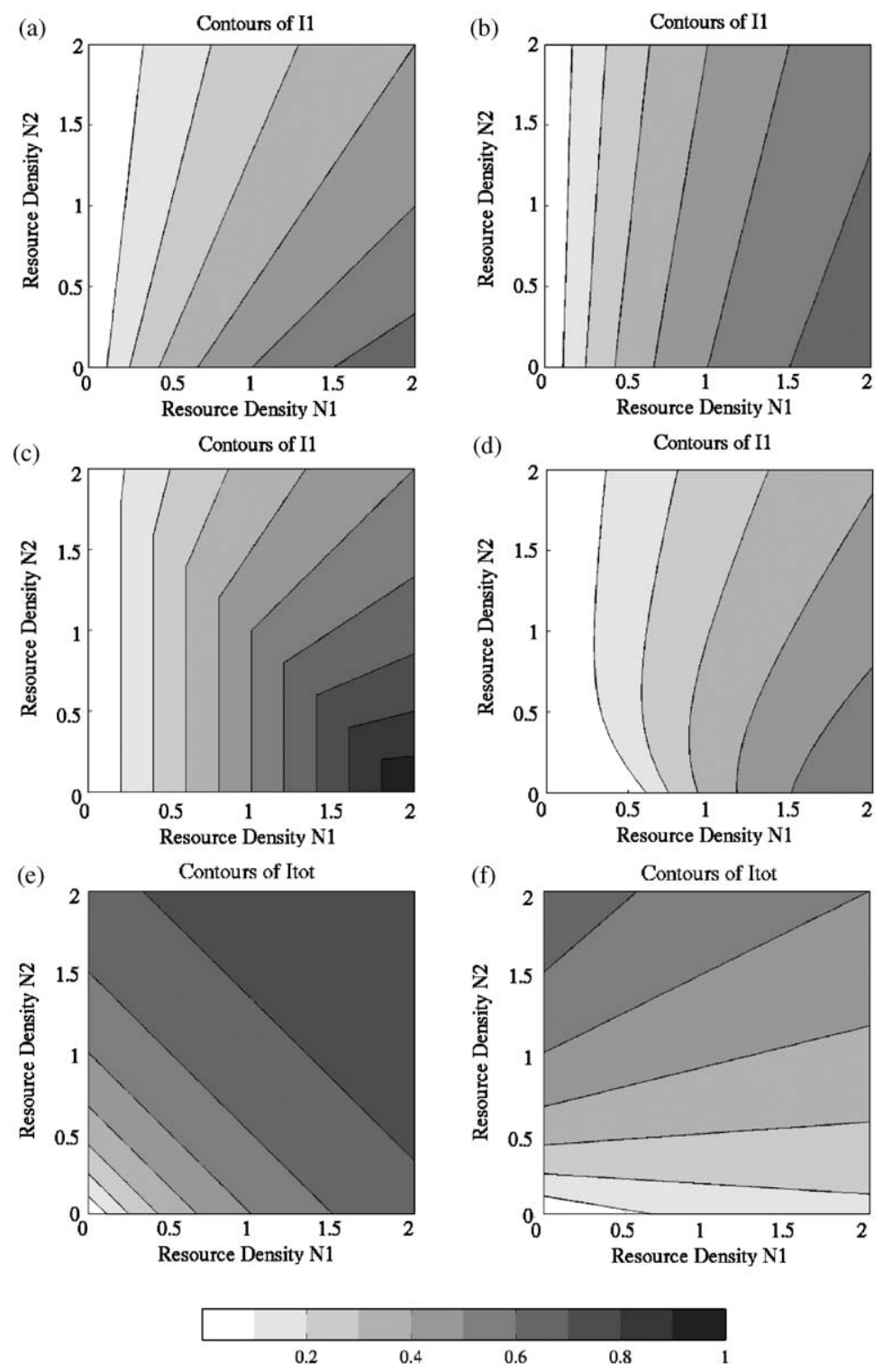

Fig. 3. Class 1 multiple resource functional responses. Contour plots of nutritional intake from two resources versus resource densities $\left(N_{1}\right.$ and $\left.N_{2}\right)$ for Class 1: No Switching examples (see text and Table 2a for model descriptions). (a) Disk/Michaelis-Menten $I_{1}$, equal preferences $\left(a_{1}=a_{2}=1, h_{1}=h_{2}=1\right.$; equivalent to $\left.m_{1}=m_{2}=1, k=1, p_{1}=p_{2}=1\right)$; (b) Disk/Michaelis-Menten $I_{1}$, unequal preferences $\left(a_{1}=1, a_{2}=0.25, h_{1}=h_{2}=1\right.$; equivalent to $\left.m_{1}=m_{2}=1, k=1, p_{1}=1, p_{2}=0.25\right)$; (c) Rectilinear $I_{1}$, equal preferences $\left(m=1, v=2, p_{1}=p_{2}=1\right)$; (d) Threshold $I_{1}$, equal preferences $\left(m, k=1, p_{1}=p_{2}=1, \tau=0.5\right)$; (e) Disk/Michaelis-Menten $I_{\text {tot }}$, parameters as in (a); (f) Disk/Michaelis-Menten $I_{\text {tot }}$, unequal preferences from unequal handling times $/$ maximum rates $\left(a_{1}=a_{2}=1\right.$, $h_{1}=4, h_{2}=1$; equivalent to $\left.m_{1}=0.25, m_{2}=1, k=1, p_{1}=p_{2}=1\right)$.

and multiple resource responses, although this is not obvious in the overparameterized versions. For example, it may incorrectly appear that active selection is assumed by the Michaelis-Menten model because $k_{i}^{*}$, the half-saturation constant of $I_{i}^{* i m p}$, is generally not equal to $k$, the so-called "half-saturation constant" of $I_{i}$ (i.e. $k_{i}^{*}=k / p_{i}$ ). However, $k$ and $k_{i}^{*}$ should not be directly 
compared because the two have different biological significance; $k$ is related to weighted, not actual, resource densities (Eq. (14)). The same is true for the feeding thresholds $\tau$ and $\tau_{i}^{*}$ in the Threshold model.

Analysis of $I_{i}^{* \text { imp }}$ for the Michaelis-Menten, Threshold, Ivlev, and Rectilinear models further reveals that $P_{i}$ of their reduced-parameter versions are both measurable and meaningful quantities. In contrast, $p_{i}$ of their overparameterized versions can only be determined when $P_{i}$ are known a priori. For example, the Michaelis-Menten $P_{i}$ are the reciprocal of $k_{i}^{*}$, whereas $p_{i}$ are set by the actual $k_{i}^{*}$ once the modeler chooses a value for $k$. When the relationship between $k_{i}^{*}, k$, and $p_{i}$ is unrecognized, modelers unwittingly assume specific values for the single resource half-saturation constants. Hence, not only is the overparameterization of these models unjustified, but it can obfuscate interpretation of behavior and choice of appropriate parameter values.

\subsubsection{Diagnostic III}

Like their single resource analogs, the multiple resource Disk/Michaelis-Menten and Ivlev $I_{i}$ always increases when $N_{i}$ increases, regardless of resource preferences (Table 2b, Fig. 3a-b). While the Rectilinear model exhibits the same general dynamic, the rate of change decreases sharply once intake is maximal, and intake never satiates on any one resource, which is in contrast to its single resource analog (Fig. 3c). The Threshold $I_{i}$ only increases with $N_{i}$ where resource densities are sufficiently high; variations in $N_{i}$ are assumed to have no effect where resource densities are low (Fig. 3d).

\subsubsection{Diagnostic IV}

The Disk/Michaelis-Menten and Ivlev models always assume interference of other resources, regardless of resource preferences (Table $2 \mathrm{~b}$, Fig. 3a-b). The Rectilinear and Threshold $I_{i}$ decrease for increasing $N_{j}(j \neq i)$ only when resource densities are high. When resource densities are low, $N_{j}$ is assumed to have no effect on the Rectilinear $I_{i}$ (Fig. 3c), whereas the Threshold $I_{i}$ increases when $N_{j}$ increases (Fig. 3d). The synergistic effect in the Threshold model arises because feeding is only assumed to cease when a weighted measure of the total resource density is less than $\tau$, so consumption of resource $i$ can occur when $N_{i}<\tau$ and even when $N_{i}<\tau_{i}^{*}$, the implied single resource threshold (Table 2b). Analysis of Diagnostic IV therefore reveals that $\tau$ is related to minimal nutritional requirements as opposed to minimal densities required for detection or attack. Therefore, the Threshold response could represent suspension-feeders or foragers that only have the energy to generate feeding currents or successfully attack resources when there sufficient total nutrition available.

\subsubsection{Diagnostic $V$}

All Class 1 examples assume feeding is always optimal when maximum rates $m_{i}$ (handling times $h_{i}$ ) are identical for all resources (Table 2b, Fig. 3e). However, feeding is sub-optimal when $m_{i}\left(h_{i}\right)$ are resource-dependent and resource densities are high, because $I_{\text {tot }}$ decreases for increases in the relative density of resources with lower $m_{i}$ (longer $h_{i}$ ) (Fig. 3f).

\subsubsection{Diagnostic VI}

All Class 1 examples assume generalism is the better strategy in regions where $I_{\text {tot }}$ increases with increasing $N_{i}$ (Table 2b, Fig. 3e). However, where feeding is sub-optimal, specialism on resources with the largest maximum rates (shortest handling times) is more nutritionally advantageous (Fig. 3f).

\subsubsection{Diagnostic VII}

All the Class 1 examples assume there is neither nutritional cost nor benefit to selecting among functionally equivalent resources (Table 2b, Fig. 3e).

\subsection{Examples of Class 2: Passive Switching models}

Examples of Class 2 models and their associated references are listed in Table 3a.The No-Interference model assumes the multiple resource functional response for each resource is the same as when it is the only available nutrition (i.e. $\left.I_{i}=I_{i}^{*}\right)$. The Modified-Threshold model, which we developed as an alternative to the Class 1 Threshold model, allows for resource-dependent maximum rates $m_{i}$ and feeding thresholds $\tau_{i}$ 
Table 3a

Class 2 multiple resource functional responses

\begin{tabular}{llll}
\hline $\begin{array}{l}\text { Class } 2 \\
\text { Formulation }\end{array}$ & Intake of resource $N_{i}$ & $\begin{array}{l}\text { Parameter } \\
\text { Dimensions }\end{array}$ & $\begin{array}{l}\text { Sample } \\
\text { References }\end{array}$ \\
\hline (A) No-Interference & $\begin{array}{l}I_{i}=I_{i}^{*} \\
\text { where } I_{i}^{*} \text { is the single resource intake from } N_{i}\end{array}$ & $\begin{array}{l}\text { dictated by } I^{*} \text { (Table 1) } \\
\text { Leonard et al. } \\
\text { (1999): } I^{*}=\text { Alternative } \\
\text { Type: Modified-Ivlev }\end{array}$ \\
\hline (B) Modified-Threshold & $I_{i}=\frac{P_{i} N_{i, \text { eff }}}{1+R} m_{i}$, where $R=\sum_{r=1}^{n} P_{r} N_{r, \text { eff }}$ and & $\begin{array}{l}{[m]=1 / T} \\
{\left[P_{i}\right]=1 /[N]} \\
{[\tau]=[N]}\end{array}$ & This paper \\
& $N_{i, \text { fff }}= \begin{cases}N_{i}-\tau_{i}, \text { for } N_{i} \geqslant \tau_{i} \\
\text { for } N_{i}<\tau_{i}\end{cases}$ &
\end{tabular}

\section{(C) Sigmoidal I} (from Disk) (from MichaelisMenten)

$$
\begin{array}{rlrl}
I_{i} & =\frac{\hat{a}_{i} N_{i}}{1+\sum_{r=1}^{n} \hat{a}_{r} h_{r} N_{r}}, \text { where } \hat{a}_{i}=c_{i} N_{i} & {\left[c_{i}\right]=1 /\left([N]^{2} T\right)} \\
& =\frac{\hat{p}_{i} N_{i}}{k^{2}+R} m_{i}, \text { where } R=\sum_{r=1}^{n} \hat{p}_{r} N_{r} \text { and } \hat{p}_{i}=p_{i} N_{i} & {\left[h_{i}\right]=[T]} & {[k]=[N]} \\
& =\frac{\hat{P}_{i} N_{i}}{1+\hat{R}} m_{i}, \quad \text { where } \hat{R}=\sum_{r=1}^{n} \hat{P}_{r} N_{r} & {\left[p_{i}\right]=\mathrm{N} . \mathrm{D} .} \\
& {\left[P_{i}\right]=1 /[N]}
\end{array}
$$

Gismervik and Andersen (1997), Edwards (2001)

where $m_{i}=1 / h_{i}, \quad \hat{P}_{i}=\hat{p}_{i} / k^{2}=\hat{a}_{i} h_{i}$

(D) Sigmoidal II

$$
\begin{array}{lll}
I_{i}=\frac{\hat{a}_{i} N_{i}}{{ }_{1+\sum_{r=1}^{n} \hat{a}_{r} h_{r} N_{r}}} \quad \text { where } \hat{a}_{i}=\frac{f_{i} N_{i}}{\left(1+g_{i} N_{i}\right)} & & {\left[f_{i}\right]=1 /\left([N]^{2} T\right)} \\
& {\left[h_{i}\right]=[T]} \\
& {\left[g_{i}\right]=1 /[N]}
\end{array}
$$

(E) Abundance-Based I

$$
\begin{aligned}
I_{i} & =\frac{\hat{p}_{i} N_{i}}{k+R} m, \quad \text { where } R=\sum_{r=1}^{n} \hat{p}_{r} N_{r} \\
\hat{p}_{i} & =\left\{\begin{array}{ll}
1-w_{i} N_{i}, & \text { for } N_{i}<\eta \\
p_{i}, & \text { for } N_{i} \geqslant \eta
\end{array} \text { and } w_{i}=\frac{\left(1-p_{i}\right)}{\eta}\right.
\end{aligned}
$$

$$
\begin{aligned}
& {\left[m_{i}\right]=1 / T} \\
& {[k]=[N]} \\
& {\left[p_{i}\right]=\text { N.D. }} \\
& {[\eta]=[N]} \\
& {\left[w_{i}\right]=1 /[N]}
\end{aligned}
$$

(Table 3a). The other Class 2 models are derived by extending the Class 1 Disk model assuming the attack rate for resource $i$ depends upon its density (i.e. the constant $a_{i}$ is replaced by $\hat{a}_{i}$ that depends on $N_{i}$ ), but the handling time $h_{i}$ remains constant. This is equivalent to extending the MichaelisMenten equation by replacing the constant $p_{i}$ with $\hat{p}_{i}$ that depends on $N_{i}$, but keeping maximum rates $m_{i}$ constant. These three models are distinguished by their assumed density-dependence: (i) linear in the first Sigmoidal model ("Sigmoidal I" in Table 3a); (ii) hyperbolic in the second Sigmoidal model ("Sigmoidal II" in Table 3a); and (iii) rectilinear in the Abundance-Based model ("Abundance-Based I" in Table 3a). The Abundance-Based model additionally assumes all $m_{i}$ are equal. All formulations based on the overparameterized Michaelis-
Menten equation also use one more parameter than is necessary to describe the functional response.

\subsection{Dynamics assumed in Class 2: Passive Switching examples}

\subsubsection{Diagnostic I}

The No-Interference $E_{i}$ equal the single resource clearance rates, $F_{i}^{*}$, which results in switching unless $I_{i}^{*}$ is Type 1 Non-Satiating. The Sigmoidal and Abundance-Based $E_{i}$ are the density-dependent analogs of Class 1 Disk/Michaelis-Menten models upon which they were based (i.e. $E_{i}=\hat{a}_{i}=$ $m_{i} \hat{P}_{i}$ or $\left.E_{i}=m_{i} \hat{p}_{i}\right)$, and the Modified-Threshold $E_{i}$ additionally depend on how $N_{i}$ scales with the threshold $\tau_{i}$ (Table 3b). All these examples assume 
Table $3 b$

Diagnostics of Class 2 examples

\begin{tabular}{|c|c|c|c|c|c|c|c|}
\hline & $\begin{array}{l}\mathrm{I} \\
E_{i}\end{array}$ & $\begin{array}{l}\text { II } \\
I_{i}^{* \mathrm{imp}}\end{array}$ & $\begin{array}{l}\text { III } \\
\partial I_{i} / \partial N_{i}\end{array}$ & $\begin{array}{l}\text { IV } \\
\partial I_{i} / \partial N_{j}\end{array}$ & $\begin{array}{l}\mathrm{V} \\
\partial I_{\mathrm{tot}} / \partial N_{i}\end{array}$ & $\begin{array}{l}\text { VI } \\
B_{\text {gen }}\end{array}$ & $\begin{array}{l}\mathrm{VII} \\
C_{\text {sel }}\end{array}$ \\
\hline $\begin{array}{l}\text { (A) No- } \\
\text { Interference }\end{array}$ & $F_{i}^{*}$ & $I_{i}^{*}$ & $\begin{array}{l}\geqslant 0 \\
\text { always } \\
\text { unless } \\
\text { Type } 4\end{array}$ & $=0$ always & $\begin{array}{l}\geqslant 0 \text { always } \\
\text { unless Type } 4\end{array}$ & $\geqslant 0$ always & $\begin{array}{l}<0,0 \text { or }>0 \\
\text { depends on } I^{*}\end{array}$ \\
\hline $\begin{array}{l}\text { (B) Modified- } \\
\text { Threshold }\end{array}$ & $\begin{array}{l}\text { for } N_{i} \geqslant \tau_{i}: \\
\qquad P_{i} m_{i} \frac{\left(N_{i}-\tau_{i}\right)}{N_{i}} \\
\text { for } N_{i}<\tau_{i}: 0\end{array}$ & $\begin{array}{l}\text { for } N_{i} \geqslant \tau_{i} \text { : } \\
\qquad \frac{\left(N_{i}-\tau_{i}\right)}{k_{i}^{*}+N_{i}-\tau_{i}} m_{i} \\
\quad \text { where } k_{i}^{*}=\frac{1}{P_{i}} \\
\text { for } N_{i}<\tau_{i}\end{array}$ & $\begin{array}{l}\geqslant 0 \\
\text { always }\end{array}$ & $\leqslant 0$ always & $\begin{array}{l}\text { equal } \\
m_{i} \geqslant 0 \\
\text { always } \\
m_{i}<m_{j}<0 \\
\text { at high } N_{j}\end{array}$ & $\begin{array}{l}\text { equal } m_{i} \geqslant 0 \\
m_{i}<m_{j}:<0 \\
\text { at high } N_{j} \text { for } \\
\text { resource } j\end{array}$ & $>0$ always \\
\hline $\begin{array}{l}\text { (C) Sigmoidal I } \\
\text { (from disk) } \\
\text { (from } \\
\text { Michaelis-- } \\
\text { Menten) }\end{array}$ & $\begin{array}{l}\hat{a}_{i} \\
\hat{p}_{i} m_{i}\left(\text { or } \hat{P}_{i} m_{i}\right)\end{array}$ & $\begin{array}{l}\frac{\hat{a}_{i} N_{i}}{1+\hat{a}_{i} h_{i} N_{i}} \\
\frac{N_{i}^{2}}{\left(k_{i}^{*}\right)^{2}+N_{i}^{2}} m_{i} \\
\text { where } k_{i}^{*}=\frac{k}{p_{i}}=\frac{1}{\sqrt{P_{i}}}\end{array}$ & $\begin{array}{l}>0 \\
\text { always }\end{array}$ & $<0$ always & $\begin{array}{l}\text { equal } h_{i} \text { or } \\
\text { equal } m_{i} \geqslant 0 \\
\text { always } \\
h_{i}>h_{j} \text { or } \\
m_{i}<m_{j}: \\
<0 \text { at high } N_{j}\end{array}$ & $\begin{array}{l}\text { equal } h_{i} \text { or } \\
\text { equal } m_{i}: \\
\geqslant 0 \\
h_{i}>h_{j} \text { or } \\
m_{i}<m_{j}:<0 \\
\text { at high } N_{j} \text { for } \\
\text { resource } j\end{array}$ & $>0$ always \\
\hline (D) Sigmoidal II & $\hat{a}_{i}$ & $\begin{array}{l}\frac{N_{i}^{2}}{\left(k_{i, 1}^{*}+N_{i}\right)\left(k_{i, 2}^{*}+N_{i}\right)} m_{i}^{*} \\
\text { where } m_{i}^{*}=\frac{1}{h_{i}}, \\
k_{i, 1}^{*}=\frac{1}{2}\left(x_{i}+\sqrt{x_{i}^{2}-4 y_{i}}\right) \\
k_{i, 2}^{*}=\frac{1}{2}\left(x_{i}-\sqrt{x_{i}^{2}-4 y_{i}}\right) \\
x_{i}=\frac{g_{i}}{f_{i} h_{i}} \text { and } y_{i}=\frac{1}{f_{i} h_{i}}\end{array}$ & $>0$ always & $<0$ always & $\begin{array}{l}\text { equal } h_{i}: \geqslant 0 \\
\text { always } \\
h_{i}>h_{j}: \\
<0 \text { at high } N_{j}\end{array}$ & $\begin{array}{l}\text { equal } \\
h_{i}: \geqslant 0 \\
h_{i}>h_{j}: \\
<0 \text { at high } N_{j} \text { for } \\
\text { resource } j\end{array}$ & $>0$ always \\
\hline $\begin{array}{l}\text { (E) Abundance- } \\
\text { Based I }\end{array}$ & $\hat{p}_{i}$ & $\begin{array}{l}\text { for } N_{i} \geqslant \eta: \\
\quad \frac{N_{i}}{k_{i}^{*}+N_{i}} m \\
\quad \text { where } k_{i}^{*}=\frac{k}{p_{i}} \\
\text { for } N_{i}<\eta: \\
\quad \frac{N_{i}-w_{i} N_{i}^{2}}{k+N_{i}-w_{i} N_{i}^{2}} m\end{array}$ & $\begin{array}{l}<0 \text { at } \\
\text { intermediate } \\
\text { dens. } \\
\text { depends } \\
\text { on params. }\end{array}$ & $\begin{array}{l}>0 \text { at } \\
\text { intermediate } \\
\text { dens. } \\
\text { depends } \\
\text { on params. }\end{array}$ & $\begin{array}{l}<0 \text { at } \\
\text { intermediate } \\
\text { dens. } \\
\text { depends } \\
\text { on params. }\end{array}$ & $\begin{array}{l}<0,0 \text { or } 0 \text { depends on } \\
\text { dens. and params. }\end{array}$ & $\begin{array}{l}<0,0 \text { or }>0 \\
\text { depends on dens. } \\
\text { and params. }\end{array}$ \\
\hline
\end{tabular}



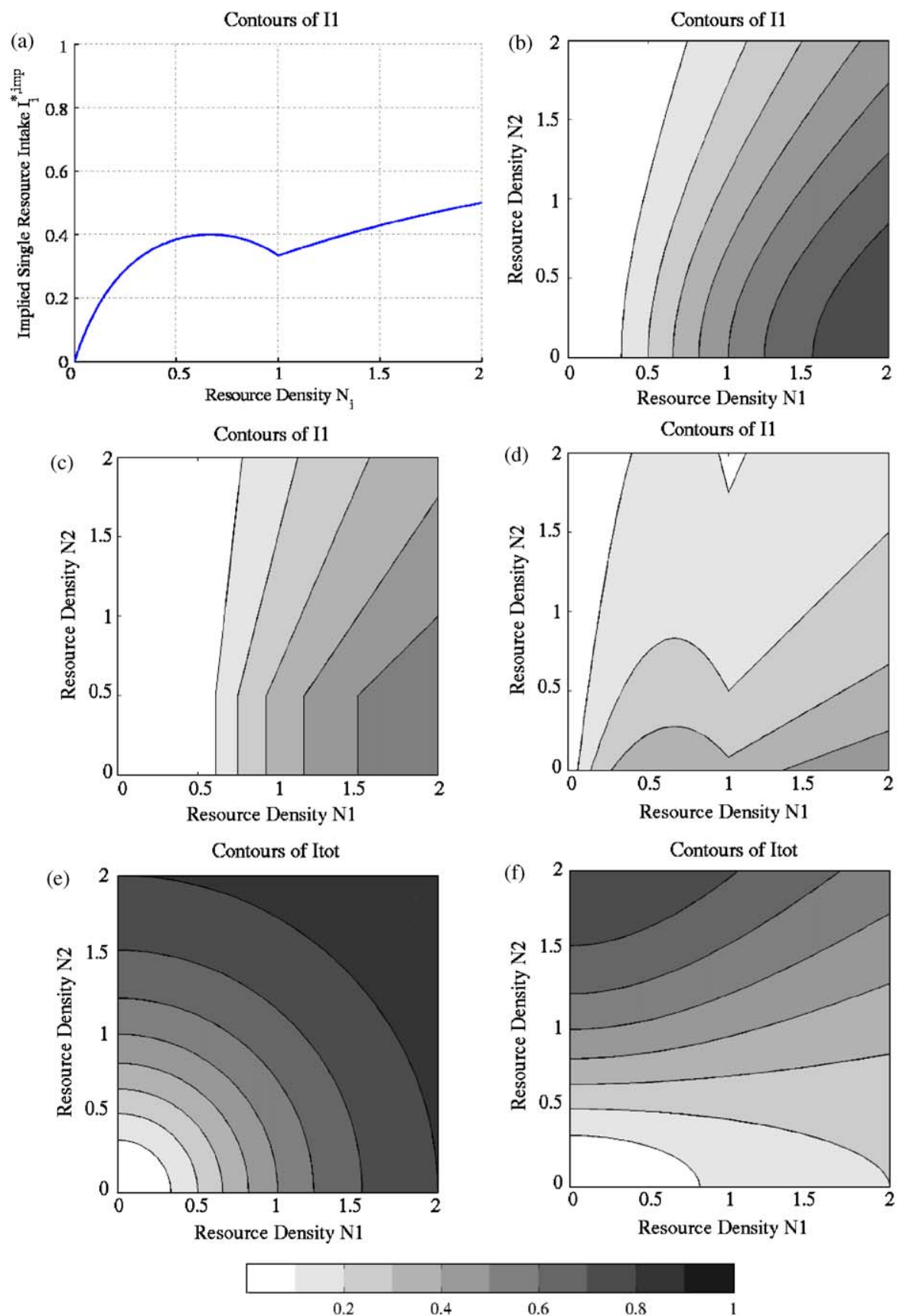

Fig. 4. Class 2 multiple resource functional responses. Contour plots of nutritional intake from two resources versus resource densities $\left(N_{1}\right.$ and $\left.N_{2}\right)$ for Class 2: Passive Switching examples (see text and Table 3a for model descriptions). "PDD" = preference density dependence. (a) Abundance-Based $I_{i}^{* i m p},\left(m=1, k=0.5, p_{i}=0.5, \eta=1\right)$; (b) Sigmoidal I $I_{1}$, equal PDD $\left(h_{1}=h_{2}=1, c_{1}=c_{2}=1\right.$; equivalent to $\left.m_{1}=m_{2}=1, k=1, p_{1}=p_{2}=1\right)$; (c) Modified-Threshold $I_{1}$, equal $\operatorname{PDD}\left(m_{1}=m_{2}=1, k=1, p_{1}=p_{2}=1\right.$, $\left.\tau_{1}=\tau_{2}=0.5\right)$; (d) Abundance-Based $I_{1}$, unequal PDD ( $\left.m=1, k=0.5, p_{1}=1, p_{2}=0.25, \eta=1\right)$; (e) Sigmoidal I $I_{\text {tot }}$, parameters as per (d); (f) Sigmoidal I $I_{\text {tot }}$, unequal PDD from unequal handling times/maximum rates $\left(h_{1}=4, h_{2}=1, c_{1}=0.25, c_{2}=1\right.$ equivalent to $\left.m_{1}=0.25 m_{2}=1, k=1, p_{1}=p_{2}=1\right)$. 
switching, since their $E_{i}$ are density-dependent. However, most examples also assume (essentially) no switching when resource densities are high. Thus, when zooplankton behavior is consistent with these models, measured clearance rates will not reveal switching unless experiments are conducted over a sufficiently broad range of densities. Furthermore, because these models' $E_{i}$ depend only upon $N_{i}$, determination of the passive nature of this switching (i.e. the reason they are Class 2) requires examination of Diagnostic II.

\subsubsection{Diagnostic II}

The No-Interference model allows for resourcedependent Types of single resource responses (e.g. Type 1 Rectilinear for one resource and Type 3 Sigmoidal for another), and the Modified-Threshold $I_{i}^{* \text { imp }}$ can also be different Types depending on whether a feeding threshold is specified (i.e. either Type 3 Threshold or Type 2 Michaelis-Menten). In contrast, the Sigmoidal and Abundance-Based $I_{i}^{* \text { imp }}$ are the same Type for all resources. Furthermore, certain parameter values result in the Abundance-Based $I_{i}^{* \text { imp }}$ being uncharacteristic of any known response (Table 3b, Fig. 4a). The behaviors and parameters are consistent between the single and multiple resource formulations for all the Class 2 examples (Table 3b). Thus, passive selection is assumed, and switching can be predicted when the actual $I^{*}$ are known. However, this fact is not obvious in the overparameterized formulations, and their extra degree of freedom is unjustified.

\subsubsection{Diagnostic III}

$I_{i}$ always increases when $N_{i}$ increases in both Sigmoidal models (Fig. 4b). The same dynamic is assumed by the Modified-Threshold model when $N_{i}>\tau_{i}$ (Fig. 4c), and by the No-Interference model when none of the single resource responses are Type 4. However, the Abundance Based $I_{i}$ can decrease when $N_{i}$ increases for certain parameter values (Fig. 4d), which results in negative switching.

\subsubsection{Diagnostic $I V$}

Other resources have no effect on the NoInterference $I_{i}$, whereas interference is always assumed for Sigmoidal $I_{i}$ (Fig. 4b). The Modified-Threshold model assumes no effect when $N_{j}(j \neq i)$ are low and interference when $N_{j}>\tau_{j}$ (Fig. 4c). In contrast, the certain parameter values result in the Abundance-Based $I_{i}$ exhibiting synergism (Table $3 \mathrm{~b}$ ), which results in regions of negative switching.

\subsubsection{Diagnostic $V$}

Feeding is always optimal in the No-Interference model, provided none of the single resource responses are Type 4 . When all maximum rates $m_{i}$ (handling times $h_{i}$ ) are equal, the ModifiedThreshold and both Sigmoidal $I_{\text {tot }}$ also always increase with increasing $N_{i}$ (Table 3b, Fig. 4e). However, these models assume feeding can be suboptimal when $m_{i}\left(h_{i}\right)$ are resource-dependent and resource densities are high (Fig. 4f). Sub-optimal feeding can occur at intermediate resource densities when certain parameter values are used in the Abundance-Based model (Table 3b).

\subsubsection{Diagnostic VI}

All Class 2 examples assume generalism is the best strategy where $I_{\text {tot }}$ increases with increasing $N_{\text {tot }}$ (Table $3 b$, Fig. 4e). However, specialism is more nutritionally advantageous where feeding is sub-optimal (i.e. high resource densities for the Modified-Threshold and both Sigmoidal models; intermediate densities for Abundance-Based).

\subsubsection{Diagnostic VII}

The Modified-Threshold and both Sigmoidal models assume there is a nutritional cost to selecting among functionally equivalent resources (Table 3b, Fig. 4e). In contrast, it can be nutritionally beneficial to distinguish among such resources in the No-Interference and AbundanceBased models, depending on $I^{*}$ and parameter values.

\subsection{Examples of Class 3: Active Switching models}

Examples of Class 3 models and associated references are listed in Table 4a. The ProportionBased model was used in Fasham et al. (1990), a planktonic ecosystem model cited hundreds of times in the literature, which suggests that this is 
Table $4 \mathrm{a}$

Class 3 multiple resource functional responses

\begin{tabular}{|c|c|c|c|}
\hline $\begin{array}{l}\text { Class } 3 \\
\text { Formulation }\end{array}$ & Intake of resource $N_{i}$ & $\begin{array}{l}\text { Parameter } \\
\text { Dimensions }\end{array}$ & References \\
\hline $\begin{array}{l}\text { (A) Proportion- } \\
\text { Based }\end{array}$ & $\begin{array}{l}I_{i}=\frac{\hat{p}_{i} N_{i}}{k+R} m, \quad \text { where } R=\sum_{r=1}^{n} \hat{p}_{r} N_{r} \\
\text { and } \hat{p}_{i}=\frac{p_{i} N_{i}}{\sum_{r=1}^{n} p_{r} N_{r}}\end{array}$ & $\begin{array}{l}{\left[m_{i}\right]=1 / T} \\
{[k]=[N]} \\
{\left[p_{i}\right]=\text { N.D. }}\end{array}$ & $\begin{array}{l}\text { Fasham et al. (1990), } \\
\text { Fasham et al. (1993), } \\
\text { Chai et al. (1996), } \\
\text { Loukos et al. (1997), } \\
\text { Strom and Loukos (1998), } \\
\text { Pitchford and Brindley (1999) }\end{array}$ \\
\hline $\begin{array}{l}\text { (B) Abundance- } \\
\text { Based II }\end{array}$ & $\begin{array}{l}I_{i}=\frac{\hat{p}_{i} N_{i}}{k+R} m, \quad \text { where } R=\sum_{r=1}^{n} \hat{p}_{r} N_{r} \\
\text { and } \hat{p}_{i}=\left\{\begin{array}{ll}1-\tilde{N} w_{i} & \text { for } \tilde{N}<\eta \\
p_{i} & \text { for } \tilde{N} \geqslant \eta\end{array}, \quad w_{i}=\frac{\left(1-p_{i}\right)}{\eta}\right. \\
\text { and } \tilde{N} \text { is resource with largest } p_{i}\end{array}$ & $\begin{array}{l}{\left[m_{i}\right]=1 / T} \\
{[k]=[N]} \\
{\left[p_{i}\right]=\mathrm{N} . \mathrm{D}} \\
{[\eta]=[N]} \\
{\left[w_{i}\right]=1 /[N]}\end{array}$ & Strom and Loukos (1998) \\
\hline $\begin{array}{l}\text { (C) Modified- } \\
\text { Disk }\end{array}$ & $\begin{array}{l}I_{i}=\frac{\hat{a}_{i} N_{i}}{1+\sum_{r=1}^{n} \hat{a}_{r} \hat{h}_{r} N_{r}}, \text { where } \\
\hat{a}_{i}=a_{i}+\sum_{\substack{j=1 \\
j \neq 1}}^{n} A_{i j} N_{j} \text { and } \hat{h}_{i}=h_{i}+\sum_{\substack{j=1 \\
j \neq 1}}^{n} H_{i j} N_{j}\end{array}$ & $\begin{array}{l}{\left[a_{i}\right]=1 /([N] T)} \\
{\left[A_{i j}\right]=1 /\left([N]^{2} T\right)} \\
{\left[h_{i}\right]=[T]} \\
{\left[H_{i j}\right]=T /[N]}\end{array}$ & Colton (1987) \\
\hline $\begin{array}{l}\text { (D) Modified- } \\
\text { Michaelis-Menten }\end{array}$ & $\begin{array}{l}I_{i}=\frac{N_{i}}{k+\sum_{r=1}^{n} N_{r}} m, \\
=\frac{N_{i}}{k_{i, \mathrm{eff}}+N_{i}} m \text { where } k_{r, \mathrm{eff}}=k_{i}+\sum_{r \neq i} N_{r} \\
\text { and at least two } k_{i} \text { are different } \\
\text { (otherwise this model reduces } \\
\text { to Class 1 Michaelis-Menten with } \\
\text { equal preferences) }\end{array}$ & $\begin{array}{l}{\left[m_{i}\right]=1 / T} \\
{\left[k_{i}\right]=[N]}\end{array}$ & Moloney and Field (1991) \\
\hline (E) Switching & $\begin{array}{l}I_{i}=\rho_{i, \mathrm{eff}} N_{i}, \quad \text { where } \rho_{i, \mathrm{eff}}=\frac{b_{i} N_{i}^{\lambda}}{R} \rho_{i} \\
\text { and } R=\sum_{r=1}^{n} b_{r} N_{r}^{\lambda}\end{array}$ & $\begin{array}{l}{\left[\rho_{i}\right]=1 /([N] T)} \\
{[\lambda]=\text { N.D. }} \\
{\left[b_{i}\right]=\text { N.D. }}\end{array}$ & $\begin{array}{l}\text { Tansky (1978), } \\
\text { Matsuda et al. (1986) }\end{array}$ \\
\hline $\begin{array}{l}\text { (F) Weighted- } \\
\text { intake }\end{array}$ & $\begin{array}{l}I_{i}=I_{i}^{*} \hat{z}_{i}, \\
\text { where } I_{i}^{*}=\text { is the single resource response } \\
\text { for resource } i \text {, and } \hat{z}_{i}=\frac{z_{i} I_{i}^{*}}{\sum_{r=1}^{n} z_{r} I_{r}^{*}}\end{array}$ & {$\left[z_{i}\right]=\mathrm{N} . \mathrm{D}$} & $\begin{array}{l}\text { Pace et al. (1984): } \\
I^{*}=\text { Type } 1 \\
\text { Rectilinear with } \\
\text { additional lower } \\
\text { feeding threshold }\end{array}$ \\
\hline
\end{tabular}

perhaps the most frequently used multiple resource functional response for zooplankton. Both the Proportion-Based and the Abundance-Based ("Abundance-Based II" in Table 4a) models extend the Class 1 Michaelis-Menten equation by replacing the constant $p_{i}$ with density-dependent $\hat{p}_{i}$, and assuming maximum rates are equal for all resources. The difference from the Class 2 examples derived in a similar manner is that these two Class 3 models assume $\hat{p}_{i}$ vary with the densities of other resources $N_{j}(j \neq i)$. The Proportion-Based $\hat{p}_{i}$ depend on the relative contribution of $N_{i}$ to a weighted measure of total resource density, $R$. The Class 3 Abundance-Based $\hat{p}_{i}$ all vary with $\tilde{N}$, the density of one particular resource assigned the highest $p_{i}$, according to the rectilinear relationship described for the Class 2 AbundanceBased model. The Modified-Disk model extends the Class 1 Disk equation by assuming both attack rates $a_{i}$ and handling times $h_{i}$ vary linearly with 
Table 4b

Diagnostics of Class 3 examples

\begin{tabular}{|c|c|c|c|c|c|c|c|}
\hline & $\begin{array}{l}\mathrm{I} \\
E_{i}\end{array}$ & $\begin{array}{l}\text { II } \\
I_{i}^{* i m p}\end{array}$ & $\begin{array}{l}\text { III } \\
\partial I_{i} / \partial N_{i}\end{array}$ & $\begin{array}{l}\text { IV } \\
\partial I_{i} / \partial N_{j}\end{array}$ & $\begin{array}{l}\mathrm{V} \\
\partial I_{\mathrm{tot}} / \partial N_{i}\end{array}$ & $\begin{array}{l}\text { VI } \\
B_{\text {gen }}\end{array}$ & $\begin{array}{l}\mathrm{VII} \\
C_{\text {sel }}\end{array}$ \\
\hline $\begin{array}{l}\text { (A) Proportion- } \\
\text { Based }\end{array}$ & $p_{i} N_{i}$ & $\frac{N_{i}}{k+N_{i}} m$ & $>0$ always & $\leqslant 0$ always & $<0$ for low dens. & $\begin{array}{l}\text { only }>0 \text { for } \\
\text { high dens. }\end{array}$ & $>0$ always \\
\hline $\begin{array}{l}\text { (B) Abundance- } \\
\text { Based II }\end{array}$ & $\begin{array}{l}\text { for } \tilde{N}<\eta=1-w_{i} \tilde{N} \\
\text { for } \tilde{N} \geqslant \eta=p_{i}\end{array}$ & $\begin{array}{l}\text { for } \tilde{i}=\text { same } \\
\text { as Class } 2 \\
\text { Ab.-Based } 1 \\
\text { for } i \neq \tilde{i}=\frac{N_{i}}{1+N_{i}} m\end{array}$ & $\begin{array}{l}<0 \text { at } \\
\text { intermediate } \\
\text { dens. } \\
\text { depends } \\
\text { on params. }\end{array}$ & $\begin{array}{l}>0 \text { at } \\
\text { intermediate } \\
\text { dens. } \\
\text { depends } \\
\text { on params. }\end{array}$ & $\begin{array}{l}<0 \text { at } \\
\text { intermediate } \\
\text { dens. } \\
\text { depends on } \\
\text { params. }\end{array}$ & $\begin{array}{l}<0 \text { at } \\
\text { intermediate } \\
\text { dens. } \\
\text { depends } \\
\text { on params. }\end{array}$ & $\begin{array}{l}<0,0 \text { or }>0 \\
\text { depends on dens. } \\
\text { and params. }\end{array}$ \\
\hline $\begin{array}{l}\text { (C) Modified- } \\
\text { Disk }\end{array}$ & $\hat{a}_{i}$ & $\frac{a_{i} N_{i}}{1+a_{i} h_{i} N_{i}}$ & $\begin{array}{l}<0 \text { at low dens. } \\
\text { depends on } \\
\text { params. }\end{array}$ & $\begin{array}{l}>0 \text { at low dens. } \\
\text { depends on } \\
\text { params. }\end{array}$ & $\begin{array}{l}<0 \text { at high and low } \\
\text { dens. } \\
\text { depends on } \\
\text { params. }\end{array}$ & $\begin{array}{l}\text { only }>0 \text { at } \\
\text { low dens. }\end{array}$ & $>0$ always \\
\hline $\begin{array}{l}\text { (D) Modified- } \\
\text { Michaelis-- } \\
\text { Menten }\end{array}$ & $\frac{1}{k_{i}+\sum_{r=1}^{n} N_{r}}$ & $\frac{N_{i}}{k_{i}+N_{i}} m$ & $>0$ always & $\leqslant 0$ always & $\begin{array}{l}<0 \text { at high dens. } \\
\text { depends on params. }\end{array}$ & $\begin{array}{l}<0 \text { at high } N_{j} \\
\text { for resource } j\end{array}$ & $\begin{array}{l}\text { N/A } \\
\text { (with } \\
\text { func. equiv. } \\
\text { resources, } \\
\text { model is } \\
\text { Class } 1 \text { M.M.) }\end{array}$ \\
\hline (E) Switching & $\left(b_{i} N_{i}\right)^{\lambda} \rho_{i}$ & $\rho_{i} N_{i}$ & $>0$ always & $\leqslant 0$ always & $\begin{array}{l}<0 \text { at high and } \\
\text { low dens. } \\
\text { depends on } \\
\text { params. }\end{array}$ & $<0$ at low dens. & $>0$ always \\
\hline $\begin{array}{l}\text { (F) Weighted- } \\
\text { Intake }\end{array}$ & $I_{i}^{*} F_{i}^{*} z_{i}$ & $I_{i}^{*}$ & $\begin{array}{l}\geqslant 0 \text { always } \\
\text { unless Type } 4\end{array}$ & $\begin{array}{l}\leqslant 0 \text { always } \\
\text { unless Type } 4\end{array}$ & $\begin{array}{l}<0 \text { at high and } \\
\text { low dens. } \\
\text { depends on } \\
\text { params. }\end{array}$ & $<0$ at low dens. & $\begin{array}{l}\geqslant 0 \text { always } \\
\text { unless Type } 4\end{array}$ \\
\hline
\end{tabular}


$N_{j}(j \neq i)$, according to the constant rates of change $A_{i j}$ and $H_{i j}$ (Table $3 \mathrm{a}$ ).

The Modified-Michaelis-Menten, Switching and Weighted-Intake models are all based on assumptions about how other resources affect the single resource response, without linking the changes to any specific behavioral mechanism. The ModifiedMichaelis-Menten model assumes other resources act to increase the effective half-saturation constant $k_{i \text {,eff }}$ of a Type 2 Michaelis-Menten $I_{i}^{*}$ (Table 3a). The Switching model assumes other resources act to reduce the effective rate of change $\rho_{i, \text { eff }}$ of a Type 1 Non-Satiating, according to a weighted exponential measure of the total resource density (Table 3a). In the Weighted-Intake model, $I_{i}^{*}$ is reduced according to its relative contribution to a weighted measure of the total of all $I_{i}^{*}$, where $z_{i}$ are the weights (Table 3a).

\subsection{Dynamics assumed in Class 3: Active Switching examples}

\subsubsection{Diagnostic I}

The Class 3 Modified-Disk, Proportion-Based and Abundance-Based $E_{i}$ equal the density-dependent analogs of the Class 1 Disk/MichaelisMenten models on which they were based (i.e. $E_{i}=\hat{a}_{i}$ or $\left.E_{i}=\hat{p}_{i}\right)$, but the Modified-MichaelisMenten $E_{i}$ take a radically different form (Table 4b). The Switching and Weighted-Intake $E_{i}$ depend on both the single resource responses, $I_{i}^{*}$, and the parameters related to the assumed influence of other resources. The AbundanceBased, Modified-Disk and Modified-MichaelisMenten $E_{i}$ each depend on $N_{j}(j \neq i)$, and therefore obviously assume active switching (i.e. Class 3 ). Classification as active switching for the three other examples requires examination of Diagnostic II.

\subsubsection{Diagnostic II}

Most Class 3 examples assume the single resource responses are all the same Type (Table 4b). The Proportion-Based model further assumes all resources are functionally equivalent, in that the parameters of $I_{i}^{* \text { imp }}$ are identical for all resources. The Modified-Michaelis-Menten allows for resource-dependent single resource half-saturation constants, and the Modified-Disk allows for resource-dependent attack rates and handling times. In the Class 3 Abundance-Based model, one resource solicits a non-standard response (i.e. Fig. 4a), while the others are assumed to be functionally equivalent with half-saturation constants always equal to 1 in whatever units the resource densities are measured. In contrast, the Weighted-Intake model allows each single resource response to be any type.

Examination of $I_{i}^{* \text { imp }}$ further reveals that certain parameters of the Proportion-Based, WeightedIntake and Switching models cannot be predicted from knowledge of the single resource responses (i.e. $p_{i}, b_{i}, \lambda$ and $z_{i}$, Table $4 \mathrm{~b}$ ) Therefore, behavior in these three models is assumed to depend on the relative resource densities (i.e. active selectionthe reason they are considered Class 3), and these parameters would have to be determined through multiple resource experiments. $I_{i}^{* i m p}$ also demonstrates that the overparameterization of the Proportion-Based and Switching models is justified, because their extra degree of freedom relates to a measurable quantity. That is the multiple resource $k$ is the half-saturation constant of the single resource response in the Proportion-Based model, and $\rho_{i}$ is the single resource clearance rate in the Switching model.

\subsubsection{Diagnostic III}

The Proportion-Based $I_{i}$ always increases when $N_{i}$ increases (Table $4 \mathrm{~b}$ ), and the Weighted-Intake model makes the same assumption when none of the single resource responses are Type 4 . Of the other Class 3 examples, only the Modified-Disk and Abundance-Based models ever assume $I_{i}$ can decrease when $N_{i}$ increases, which results in regions of negative switching (Fig. 5a). This Type 4 kind of dynamic is due to the non-standard AbundanceBased $I_{i}^{* i m p}$, whereas in the Modified-Disk model it results from the assumed behavioural changes.

\subsubsection{Diagnostic $I V$}

The Proportion-Based model always assumes interference (Table 4b). Of the other Class 3 examples, only the Modified-Disk and Abundance-Based $I_{i}$ ever increase when $N_{j}$ increases 

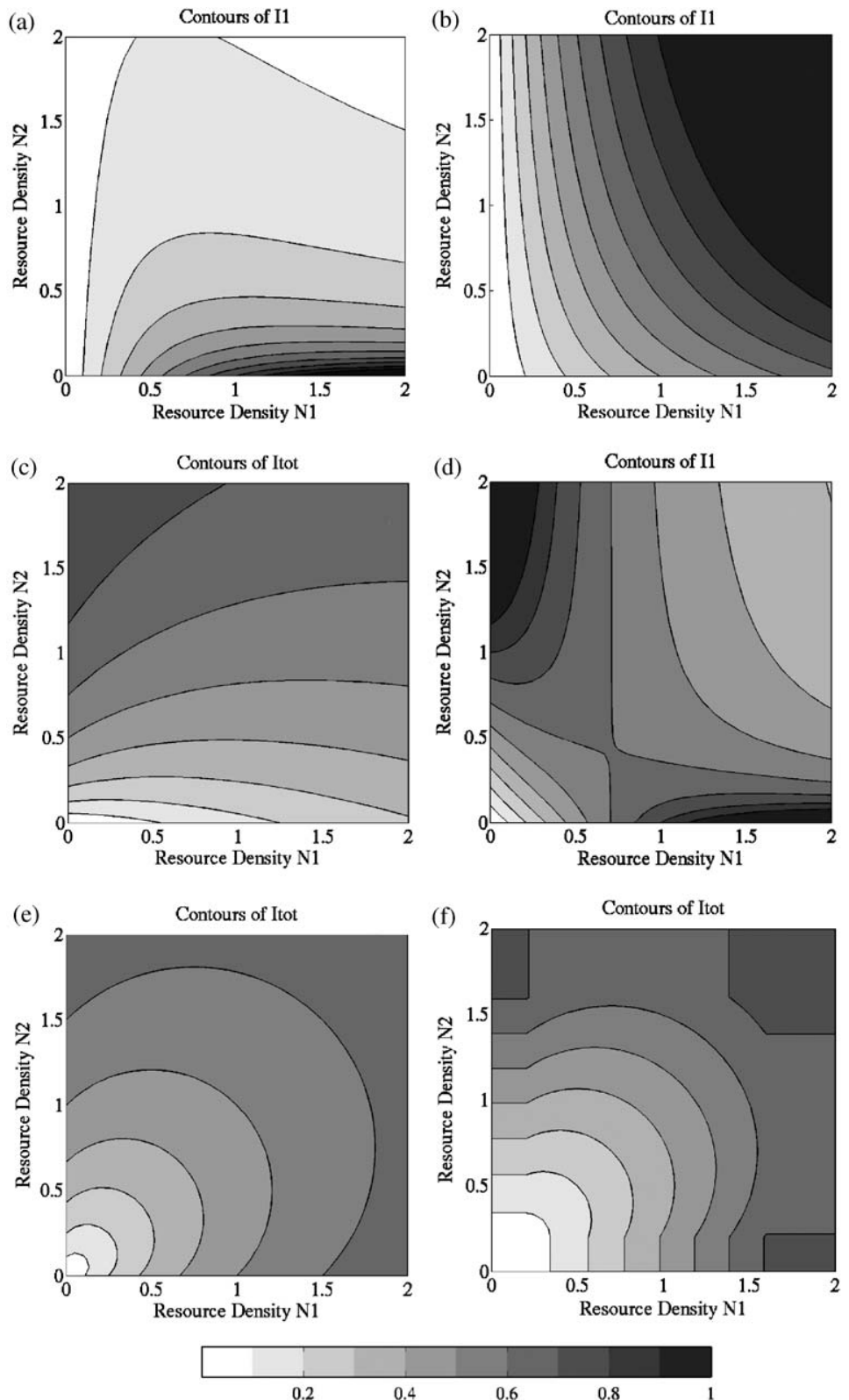

Fig. 5. Class 3 multiple resource functional responses. Contour plots of nutritional intake from two resources versus resource densities $\left(N_{1}\right.$ and $\left.N_{2}\right)$ for Class 3: Active Switching examples (see text and Table 4a for model descriptions). "PDD" = preference density dependence. (a) Modified-Disk $I_{1}$, unequal PDD with equal handling times $\left(a_{1}=a_{2}=1, A_{12}=0, A_{21}=1, h_{1}=h_{2}=0.25, H_{12}=0\right.$, $\left.H_{21}=2\right)$; (b) Modified-Disk $I_{1}$, another unequal PDD with equal handling times $\left(a_{1}=a_{2}=0.5, A_{12}=1, A_{21}=0, h_{1}=h_{2}=0.5\right.$, $\left.H_{12}=H_{21}=0\right)$; (c) Modified-Michaelis-Menten $I_{\text {tot }}$, unequal PDD with equal maximum rates $\left(m_{1}=m_{2}=1, k_{1}=5, k_{2}=0.5\right.$, $\left.p_{1}=p_{2}=1\right)$; (d) Modified-Disk $I_{\text {tot }}$, with parameters as in (a); (e) Proportion-Based $I_{\text {tot }}$, equal PDD $\left(m_{1}=m_{2}=1\right.$, $k=1, p_{1}=p_{2}=0.5$ ); (f) Weighted-Intake $I_{\text {tot }}$, equal PDD $\left(z_{1}=z_{2}=1\right)$, where $I_{i}^{*}$ is a rectilinear model (Table 1) with a lower feeding threshold, as in Pace et al. (1984). 
and results in regions of negative switching (Fig. 5b). This synergistic effect is again due to the non-standard Abundance-Based $I_{i}^{* \text { imp }}$, and the Modified-Disk's assumed behavioural changes.

\subsubsection{Diagnostic $V$}

In all the Class 3 examples, $I_{\text {tot }}$ can decrease when $N_{i}$ increases, and this assumption occurs for wider ranges of resource densities and parameter values than the passive selection models. For example, even when all maximum rates $m_{i}$ (handling times $h_{i}$ ) are equal, sub-optimal feeding occurs at high resource densities in the Modified-Michaelis-Menten and Modified-Disk models (Figs. 5c-d), at intermediate resource densities in the Abundance-Based model, and at low resource densities in the Switching and Proportion-Based models (Fig. 5e). When formulated as in Pace et al. (1984), the Weighted-Intake model also assumes sub-optimal feeding at low resource densities (Fig. 5f).

\subsubsection{Diagnostic VI}

All the Class 3 examples assume there are regions where specialism is more nutritionally advantageous than generalism (Table 4b). However, these regions are not restricted to where feeding is suboptimal. In several models, specialism is assumed to be a better strategy even when resources are functionally equivalent and/or resource densities low (e.g., Proportional-Based: Fig. 5e).

\subsubsection{Diagnostic VII}

There is a nutritional cost to selection in most Class 3 examples (Table 4b), including those assuming resources are functionally equivalent, such as the Proportion-Based model. The Abundance-Based model additionally assumes there is sometimes a benefit to distinguishing among resources.

\section{Discussion}

\subsection{Assumed dynamics and their ecological consequences}

Our review identifies published models in all three classes of multiple resource functional responses: no, passive, and active switching, although such distinctions were rarely made in the literature. The greatest differences among Class $1 I_{i}$ occurred when resource $i$ was rare, and parameter values had little influence on contour shapes. In contrast, examples in both switching classes also exhibited different dynamics for $I_{i}$ when resource $i$ was common, and contour shapes were sensitive to parameter values. The variations of $I_{i}$ versus $N_{i}$ were generally analogous to the different types of single resource responses. Most multiple resource models never assumed $I_{i}$ decreased when $N_{i}$ increased; however, this Type 4 kind of dynamic arose in some active switching examples. The assumed variation of $I_{i}$ versus $N_{j}(j \neq i)$ covered the spectrum of possible responses (i.e. no effect, interference, and synergism), even just among the Class 1 examples. Switching models generally assumed interference, but varying parameter values and/or resource densities led to negative switching in some cases.

We also found a wide diversity of modeled dynamics for $I_{\text {tot }}$, especially with respect to the optimality of feeding. Examples in all three classes assumed there were regions where $I_{\text {tot }}$ decreased when total available nutrition increased, even when none of the single resource responses were Type 4 . In the Class 1 and 2 passive selection models, such sub-optimal feeding occurred only when more nutritious resources became relatively more rare. In such circumstances, these models assumed specialization on high quality resources was a better strategy than generalism. The ActiveSwitching models demonstrated sub-optimal feeding over wider ranges of resource densities, including when resources were of equal quality. Many Class 3 models additionally assumed specialism was more nutritionally advantageous than generalism when resources were rare and zooplankton were highly food-limited, although some assumed the reverse. The No-Switching models never assumed a cost or benefit to selecting among resources of equal quality, whereas switching models generally assumed zooplankton that perceived functionally equivalent resources as a single nutrient pool would be more successful. However, some models assumed it was occasionally beneficial to distinguish among such resources. 
The different dynamics assumed for $I_{i}$ and $I_{\mathrm{tot}}$ can have vastly different ecological consequences. For example, responses for which $I_{i}$ increases with $N_{i}$ tend to have a stabilizing influence on resource dynamics, in that predation may suppress resource blooms, especially when the effective preference for resource $i$ depends on $N_{i}$. In contrast, blooms are more likely to arise where predation pressure is reduced as $N_{i}$ increases. The stability of resource $i$ is moderated by a model's assumptions regarding the effect of other resources $N_{j}(j \neq i)$. Models assuming interference decrease predation on $i$ when $j$ becomes relatively more abundant, especially when predators switch. Such responses can promote biodiversity by affording a refuge for resources that are relatively more rare. However, models assuming synergism can result in extinction of rare resources, even when this dynamic is not technically "negative switching" (e.g., Class 1 Threshold). Models assuming sub-optimal feeding may result in starved predators, whereas predators may be satiated when feeding is assumed to be optimal. As these different assumptions directly affect zooplankton growth, they indirectly affect both their ability to compete with other predators and the losses inflicted upon the resources.

\subsection{How to choose the appropriate model}

We have shown how modeling decisions can be confused by the overparameterization and/or misleading nomenclature of some multiple resource models. Ignorance about the actual response also may prompt modelers to use previously published formulations, without considering whether they are appropriate for the new application. However, as discussed above, even seemingly subtle differences in parameter values, density-dependence and/or density ranges result in drastically different dynamics. Poor model choices will incorrectly quantify resource preferences, over (or under)estimate resource consumption and predator growth, and predict contrasting effects of changing resource densities. Such misrepresentations can mislead conclusions about the importance of omnivory or magnitude of secondary production. In systems where resources and predators are tightly coupled, poor model choices also can result in spurious dynamics such as erroneously creating (or suppressing) blooms or erroneously affording resource refuges (or causing extinction).

In order for a model to be useful, its math needs to be consistent with the biology it is trying to represent. Recognition of the assumed dynamics can help modelers make appropriate choices for their application. The implied single resource response, $I_{i}^{* i m p}$, is perhaps the most important diagnostic for this assessment. It dominates the modeled response when other resource densities are low, dictates the preferences and switching in passive selection models, and determines the nature of the behavioral assumptions in active selection responses. It also can reveal problems with candidate formulations, thereby ruling them out or indicating where they need to be modified.

Certain models were shown to have $I_{i}^{* \text { imp }}$ that are uncharacteristic of any known Types (i.e. Abundance-Based for certain parameters), which recommends against their use. Inappropriate Types may also be assumed by other models, since many examples consider single resource responses to be the same type for all resources when they are generally resource-dependent. When actual single resource responses are consistent with the assumed types, $I_{i}^{* i m p}$ reveals the biological significance of the multiple resource model parameters, identifies which are more precisely known and how they relate to experimental measurements. Therefore, Diagnostic II determines whether the overparameterization occurring in some models is justified (e.g., Proportion-Based) or not (e.g., Michaelis-Menten), and indicates whether an assumption such as "equal maximum rates" is reasonable (e.g., copepod ingestion of different species or size classes: Frost, 1972; Ambler, 1986; Gismervik and Andersen, 1997; ciliate growth on algae: Stoecker et al., 1986; Verity, 1991; Montagnes, 1996), or not because resources have different handling times, nutritional quality, and/or accessibility (e.g., copepod nauplii: Ambler, 1986; ciliate growth on nanoplankton: Verity, 1991).

Diagnostics III-V are also helpful in determining whether a model is appropriate for a specific 
application. For example, while one can hypothesize reasons for a Type 4 response (e.g., toxicity or confusion) or synergistic effects of other resources (e.g., more efficient searching), there should be actual biological support for such anomalous dynamics before employing models that exhibit them (e.g., Class 1 Threshold is consistent with Calanus pacificus ingesting phytoplankton; Landry, 1981). One can similarly hypothesize reasons for sub-optimal feeding, such as energetic costs of switching (Fasham et al., 1990) or fitness being unaffected by foraging yield (Holt, 1983). However, theoretical arguments suggest natural selection would diminish the degree of suboptimality (Holt, 1983), and observational evidence indicates that predators do select more nutritious resources (Cowles et al., 1988; Verity, 1991 and references therein; Strom and Loukos, 1998, and references therein; Meyer-Harms et al., 1999). We know of no observations of decreasing nutritional intake for increasing available nutrition when resource densities are those naturally encountered by the zooplankton. This suggests that modelers should avoid use of formulations that assume sub-optimal feeding for their system's normal density ranges.

Except for the Modified-Disk, all Class 3: Active Switching models we cite are based on hypothesized-not observed-behaviors. Authors typically claimed the motivation for their assumed behavioral density-dependence was that predators would focus on resources yielding greater nutrition. However, all these active selection examples exhibit the same kind of sub-optimal feeding as the passive selection models: there are regions where total nutritional intake decreases for increasing resource density. Unlike any passive selection responses, this dynamic occurs even when resources are of the same nutritional quality, and when resource densities are low. Yet, the latter condition is where selective pressure to feed optimally would likely be greatest because nutritional yield would be critical for survival. We therefore recommend against use of any unsupported Class 3 examples, especially for regions where predators are highly food-limited (e.g. HNLC).
While any individual species may exhibit suboptimal feeding when there are dramatic changes in environmental conditions, such changes often lead to shifts in the local community structure (e.g. HNLC regions when iron is added). Zooplankton would likely adapt to long-term changes in conditions and/or new dominant species would emerge. Certainly, different regions have different dominant predators, which employ different feeding strategies and have different functional responses. Hence, use of any single formulation and/ or set of parameters is not recommended for largescale applications spanning wide ranges of resource densities and planktonic communities (e.g., global biogeochemical or climate-change models) as such approaches can systematically bias results. This can be particularly problematic for formulations assuming different kinds of dynamics for different resource densities (e.g. ProportionBased). Large-scale applications therefore may require regionally and/or temporally varying sub-models and parameters in order to represent adequately differences among planktonic communities.

\subsection{Assessing uncertainty due to assumptions}

There is often insufficient knowledge to support the choice of any one equation. Analyses of how well different models fit observations can suggest the better candidates (e.g., Carpenter et al., 1993), but consistency of a model with data does not validate assumptions because models of natural systems are insufficiently constrained (Oreskes et al., 1994). When models results hinge on unsupported assumptions they may incorrectly corroborate or nullify hypotheses and mislead future research. This is especially important for predictive models of food-limited regions, since the greatest differences among most models' dynamics occur when resource densities are low. Hence, sensitivity analyses always should be conducted in order to assess the uncertainty introduced by our ignorance.

Many sensitivity analyses are conducted by varying parameter values, usually only one at a time and often only in one direction (e.g., Evans, 1999). This is done despite the non-linearity of 
modeled processes, or the fact that different shapes of the functional response can introduce variation into model results that is at least the magnitude of variation due to uncertainty in parameter values. We have shown how changing parameters can radically change the assumed dynamics (e.g., from interference to synergism, or optimal to suboptimal feeding), meaning sensitivity to parameter values may actually indicate sensitivity to unsupported behavioral assumptions. The literature is also rich with examples of how different dynamics arise from basic choices modelers make, such as explicitly including omnivory or aggregating different resources (e.g., May 1972, 1973; Holling, 1973; Armstrong, 1994, 1999; Polis and Strong, 1996; Pahl-Wostl, 1997).

We recommend that assumptions related to the functional response be tested by varying both parameter values and model structure. Our Diagnostics can identify formulations that assume contrasting dynamics for the range of resource densities being considered, and thereby indicate which models have the greatest potential to affect results. For example, models assuming optimal feeding could approximate upper bounds on predator growth and resource consumption. These results could be compared with the lowered growth and consumption resulting from responses for which there is a nutritional cost to selection or resource refuges (e.g. Class 2 Sigmoidal). Further comparisons could be made between models that assume resources are perceived as distinct (e.g., multiple resource food webs) versus those wherein resources are perceived as a single nutrient pool (e.g., single resource food chains). Confidence in conclusions is increased when results are relatively robust to the details of the functional response. However, when the formulation is crucial (as it usually is), then the inability to make estimates with narrow ranges is an important conclusion and aids direction of future research.

\subsection{Assumed dynamics help experimentalists}

Our review found switching (and negative switching) responses that are no-switching at high and low resource densities. We also illustrated how switching could arise from a host of mechanisms, including passive selection, and how feeding behaviors may not optimize nutritional intake nor have stabilizing influences on resources. Therefore, measurements of constant or densitydependent resource preferences are insufficient to determine the nature of a functional response, especially when experiments cover only a limited range of resource densities. Our Classes and Diagnostics can aid experimental design, clarify parameters' biological significance and help interpret zooplankton behavior.

In the same way that Diagnostics I and II together determine the class of a mathematical model, classification of an actual response requires measurements of clearance rates over ranges of combinations of resource densities and knowledge of the single resource responses. Empirical fits of the latter indicate single resource behaviors (e.g., constant attack rates like Type 1 and 2, or densitydependent ones like Type 3), and hint at candidate multiple resource models. Comparison of measured and modeled preferences reveals whether behaviors depend on the availability of other resources (e.g. $I_{i}^{*}$ are Disk, but measured preferences are not attack rates). When active selection does occur, recognition of factors affecting the composition of the diet (Diagnostic I, e.g. maximum rates), and the optimality of selection (Diagnostics V-VII) can suggest nutritionally advantageous behaviors (e.g., specialism, preferential for high quality resources, etc.), which might explain the data.

Once an empirical model is developed, our Diagnostics can elucidate the biological dynamics resulting from that response. Recognition of these assumptions helps direct future research, especially when the model's implied dynamics are inconsistent with what was expected. When the observed behavior implies Type 4, synergism, or suboptimal feeding (Diagnostic III-V) at unmeasured resource densities, experiments should be performed to confirm whether such anomalous dynamics are actually exhibited or if behavioral adaptations occur. Alternatively, when unexpected dynamics occur at measured densities, the mathematical model suggests the conceptual model should be revised. Experimental investigation of selection can be further aided by determining what 
factors affect preferences (Diagnostic I), and why certain resources may be preferred even when they are less abundant than others (Diagnostic V). Diagnostics VI and VII can help formulate hypotheses, as they suggest why the feeding strategies of dominant predators vary regionally.

\subsection{Conclusions}

The Classes and Diagnostics we defined provide a framework for considering the varied behaviors and ecological implications of multiple resource functional responses. They elucidate a models' assumptions regarding resource preferences, implied single resource responses, changes in intake with changing resource densities, nutritional benefits of generalism, and nutritional costs of selection. They reveal whether or not switching can occur, the origin of switching when it does, and where responses result in anomalous dynamics such as negative switching or sub-optimal feeding.

Our review of published multiple resource models was by no means exhaustive; however, it has still emphasized how model choice can be critical. The examples we cited exhibit dramatically different dynamics, even for seemingly subtle differences among formulations. We identified equations that generally should be avoided, such as the Abundance-Based models that are uncharacteristic of any known response, and demonstrated how there is no good reason to use any overparameterized Class 1 formulation including Michaelis-Menten. We revealed how passive selection leads to sub-optimal intake when resources are of different quality (e.g. Disk and Sigmoidal models), yet all hypothesized behavioral adaptations in the Class 3 examples, including the popular Proportion-Based model, result in wider regions of anomalous dynamics. This suggests use of existing active selection models is hard to justify for many applications, and points to the need for theoreticians and experimentalists to develop more realistic formulations.

Modeling the nutritional intake for multiple resources is more complicated than it might seem. Choosing a formulation is not a straightforward; it depends on the specific zooplankton and resources being considered. Our diagnostics can assist in this determination by constraining parameters, interpreting behaviors, and recognizing limitations to a model's utility for both regional (e.g., HNLC) and large-scale applications (e.g., global biogeochemical or climate change). We identified published models with contrasting assumptions that can be used in sensitivity studies to quantify the uncertainty introduced due to ignorance about the actual response. Clarification of model dynamics also helps direct future experimental research, especially when the math is not consistent with the concept. We recommend researchers employ our framework when making decisions about multiple resource models, and thereby maximize the utility of such tools for advancing our ecological understanding and predictive capabilities.

\section{Acknowledgements}

The authors would like to thank Rob Armstrong for his comments on an early version of the paper, which greatly improved the generality and utility of this work. We would also like to acknowledge the helpful editorial feedback provided by Donald DeAngelis, Michio Kishi, and anonymous reviewer, as well as Dan Kelley, George Jackson, and Mark Kot. This work was supported by National Science Foundation US JGOFS Grant OCE-9818770.

\section{References}

Ambler, J.W., 1986. Formulations of an ingestion function for a populations of Paracalanus feeding on mixtures of phytoplankton. Journal of Plankton Research 8 (5), 957-972.

Armstrong, R.A., 1994. Grazing limitation and nutrient limitation in marine ecosystems: steady state solutions of an ecosystem model with multiple food chains. Limnology and Oceanography 39 (3), 597-608.

Armstrong, R.A., 1999. Stable model structures for representing biogeochemical diversity and size spectra in plankton communities. Journal of Plankton Research 21 (3), 445-464.

Barthel, K.G., 1983. Food uptake and growth efficiency of Eurytemora affinis (Copepoda: Calanoida). Marine Biology 74 (3), 269-274.

Bartram, W.C., 1980. Experimental development of a model for the feeding of neritic copepods on phytoplankton. Journal of Plankton Research 3 (1), 25-51. 
Campbell, R.G., Wagner, M.W., Teegarden, G.J., Boudreau, C.A., Durbin, E.G., 2001. Growth and development rates of the copepod Calanus finmarchicus in the laboratory. Marine Ecology Progress Series 221, 161-183.

Carpenter, S.R., Lathrop, R.C., Munoz-del-Rio, A., 1993. Comparison of dynamic models for edible phytoplankton. Canadian Journal of Aquatic Fisheries and Sciences 50, 1757-1767.

Chai, F., Lindey, S.T., Barber, R.T., 1996. Origin and maintenance of a high nitrate condition in the equatorial Pacific. Deep-Sea Research II 43 (4-6), 1031-1064.

Chesson, J., 1978. Measuring preference in selective predation. Ecology 59 (2), 211-215.

Chesson, J., 1983. The estimation and analysis of preference and its relationship to foraging models. Ecology 64 (5), 1297-1304.

Colton, T.F., 1987. Extending functional response models to include a second prey type: an experimental test. Ecology 68 (4), 900-912.

Cowles, T.J., Olson, R.J., Chisholm, S.W., 1988. Food selection by copepods: discrimination on the basis of food quality. Marine Biology 100, 41-49.

Davis, C.S., Flierl, G.R., Wiebe, P.H., Franks, P.J.S., 1991. Micropatchiness, turbulence and recruitment in plankton. Journal of Marine Research 49, 109-151.

Deason, E.E., 1980. Grazing of Acartia hudsonica (A. clausi) on Skeletonema costatum in Narragansett Bay (USA): influence of food concentration and temperature. Marine Biology 60 $(2 / 3), 101-113$.

DeMott, W.R., Watson, M.D., 1991. Remote detection of algae by copepods: responses to algal size, odors and motility. Journal of Plankton Research 15, 1203-1222.

Donaghay, P.L., Small, L.F., 1979. Food selection capabilities of the estuarine copepod Acartia clausi. Marine Biology 52, 137-146.

Edwards, A.M., 2001. Adding detritus to a nutrientphytoplankton-zooplankton model: a dynamical-systems approach. Journal of Plankton Research 23 (4), 389-413.

Evans, G.T., 1988. A framework for discussing seasonal succession and coexistence of phytoplankton species. Limnology and Oceanography 33 (5), 1027-1036.

Evans, G.T., 1999. The role of local models and data sets in the Joint Global Ocean Flux Study. Deep-Sea Research I 46, 1369-1389.

Fasham, M.J.R., Ducklow, H.W., McKelvie, S.M., 1990. A nitrogen-based model of plankton dynamics in the oceanic mixed layer. Journal of Marine Research 48, 591-639.

Fasham, M.J.R., Sarmiento, J.L., Slater, R.D., Ducklow, H.W., Williams, R., 1993. Ecosystem behavior at Bermuda Station 'S' and Ocean Weather Station 'India': a general circulation model and observational analysis. Global Biogeochemical Cycles 7, 379-415.

Fenchel, T., 1980. Suspension feeding in ciliated protozoa: functional response and particle size selection. Microbial Ecology 6, 1-11.
Franks, P.J.S., Wroblewski, J.S., Flierl, G.R., 1986. Behavior of a simple plankton model with food-level acclimation by herbivores. Marine Biology 91, 121-129.

Frost, B.W., 1972. Effects of size and concentration of food particles on the feeding behavior of the marine planktonic copepod Calanus pacificus. Limnology and Oceanography 17 (6), 805-815.

Frost, B.W., 1975. A threshold feeding behavior in Calanus pacificus. Limnology and Oceanography 20 (2), 263-266.

Frost, B.W., 1987. Grazing control of phytoplankton stock in the open subarctic Pacific Ocean: a model assessing the role of mesozooplankton, particularly the large calanoid copepods Neocalanus spp. Marine Ecology Progress Series 39, 49-68.

Gifford, D.J., Dagg, M.J., 1988. Feeding of the estuarine copepod Acartia tonsa Dana: carnivory versus herbivory in natural microplankton assemblages. Bulletin of Marine Science 43 (3), 458-468.

Gismervik, I., Andersen, T., 1997. Prey switching by Acartia clausi: experimental evidence and implications of intraguild predation assessed by a model. Marine Ecology Progress Series 157, 247-259.

Goldman, J.C., Dennet, M.R., Gordin, H., 1989. Dynamics of herbivorous grazing by heterotrophic dinoflagellate $O x y$ rrhis marina. Journal of Plankton Research 11 (2), 391-407.

Green, C.H., 1986. Patterns of prey selection: implications of predator foraging tactics. American Naturalist 128 (6), 824-839.

Hansen, P.J., Nielsen, T.G., 1997. Mixotrophic feeding of Fragilidium subglobosum (Dinophyceae) on three species of Ceratium: effects of prey concentration, prey species and light intensity. Marine Ecology Progress Series 147, 187-196.

Hansen, B., Tande, K.S., Bergreen, O.C., 1999. On the trophic fate of Phaeocystic pouchetii (Hariot). 3. Functional response in grazing demonstrated on juvenile stages of Calanus finmarchicus (Copepoda) fed diatoms and Phaeocystis. Journal of Plankton Research 12 (6), 1173-1187.

Hofmann, E.E., Ambler, J.W., 1988. Plankton dynamics on the outer southeastern US continental shelf. Part II: a timedependent biological model. Journal of Marine Research 40 (4), 883-917.

Holling, C.S., 1959. Some characteristics of simple types of predation and parasitism. Canadian Entomologist 91, 824-839.

Holling, C.S., 1962. Principles of insect predation. Annual Review of Entomology 6, 163-182.

Holling, C.S., 1965. The functional response of predators to prey density and its role in mimicry and population regulation. Memoirs of the Entomological Society of Canada 45, 3-60.

Holling, C.S., 1973. Resilience and stability of ecological systems. Annual Review of Ecological Systems 4, 1-24.

Holt, R.D., 1983. Optimal foraging and the form of the predator isocline. American Naturalist 122 (4), 521-541. 
Houde, S.E.L., Roman, M.R., 1987. Effects of food quality on the functional ingestion response of the copepod Acartia tonsa. Marine Ecology Progress Series 40 (1-2), 69-77.

Hutson, V., 1984. Predator mediated coexistence with a switching predator. Mathematical Biosciences 68, 233-246.

Ivlev, V.S., 1955. Experimental Ecology of the Feeding of Fishes. Pischepromizdat, Moscow, 302pp. (Translated from Russian by D. Scott, Yale University Press, New Haven, 1961.)

Jonsson, P.R., 1986. Particle size selection, feeding rates and growth dynamics of marine planktonic oligotrichous ciliates (Ciliophora: Oligotrichina). Marine Ecology Progress Series 33, 265-277.

Jonsson, P.R., Tiselius, P., 1990. Feeding behavior, prey detection, and capture efficiency of the copepod Acartia tonsa feeding on planktonic ciliates. Marine Ecology Progress Series 60, 35-44.

Jost, J.L., Drake, J.F., Tsuchiya, H.M., Fredrickson, A.G., 1973. Microbial food chains and food webs. Journal of Theoretical Biology 41, 461-484.

Kiorboe, T., Saiz, E., Viitasalo, M., 1996. Prey switching behavior in the planktonic copepod Acartia tonsa. Marine Ecology Progress Series 143, 65-75.

Lancelot, C., Hannon, E., Becquevort, S., Veth, C., De Baar, H.J.W., 2000. Modeling phytoplankton blooms and carbon export in the Southern Ocean: dominant controls by light and iron in the Atlantic sector in Austral spring 1992. Deep-Sea Research I 47, 1621-1662.

Landry, M.R., 1981. Switching between herbivory and carnivory by the planktonic marine copepod Calanus pacificus. Marine Biology 65, 77-82.

Leising, A., Gentleman, W.C., Frost, B.W., 2003. The threshold feeding response of microzooplankton within Pacific highnitrate low-chlorophyll ecosystem model under steady and variable iron input. Deep-Sea Research II, this issue (doi: 10.1016/j.dsr2.2003.07.002).

Leonard, C.L., McClain, C.R., Murtugudde, R., Hofmann, E.E., Harding Jr., L.W., 1999. An iron-based ecosystem model of the central equatorial Pacific. Journal of Geophysical Research 104 (C1), 1325-1341.

Lessard, E.J., Murrell, M.C., 1998. Microzooplankton herbivory and phytoplankton growth in the northwestern Sargasso Sea. Aquatic Microbial Ecology 16, 173-188.

Loukos, H., Frost, B., Harrison, D.E., Murray, J.W., 1997. An ecosystem model with iron limitation of primary production in the equatorial Pacific at $140^{\circ}$ W. Deep Sea Research II 44 (9-10), 2221-2249.

Matsuda, H., Kawasaki, K., Shigesada, N., Teramoto, E., Ricciardi, L.M., 1986. Switching effect on the stability of the prey-predator system with three trophic levels. Journal of Theoretical Biology 112, 251-262.

May, R.M., 1972. Limit cycles in predator-prey communities. Science 177, 900-902.

May, R.M., 1973. Stability and Complexity in Model Ecosystems. Princeton University Press, Princeton, NJ, 265pp.

May, R.M., 1977. Predators that switch. Nature 269, 103-104.
Mayzaud, P., Poulet, S.A., 1978. The importance of the time factor in the response of zooplankton to varying concentrations of naturally occurring particulate matter. Limnology and Oceanography 23, 1144-1154.

Mayzaud, P., Tirelli, V., Bernard, J.M., Roche-Mayzaud, O., 1998. The influence of food quality on the nutritional acclimation of the copepod Acartia clausi. Journal of Marine Systems 15 (1-4), 483-493.

Meyer-Harms, B., Irigoien, X., Head, R., Harris, R., 1999. Selective feeding on natural phytoplankton by Calanus finmarchicus before, during and after the 1997 spring bloom in the Norwegian Sea. Limnology and Oceanography 44 (1), 154-165.

Michaelis, L., Menten, M.L., 1913. Die Kinetik der Invertinwirkung. Biochemistry Z 49, 333-369.

Moloney, C.L., Field, J.G., 1991. Modeling carbon and nitrogen flows in a microbial plankton community. In: Reid, P.C., et al. (Ed.), Protozoa and their Role in Marine Processes, NATO ASI Series, Vol. G 25. Springer, Berlin.

Monod, J., 1942. Recherches sur la croissance des cultures bacteriennes. Hermann et Cie, Paris.

Monod, J., 1950. Annales de 1 Institut Pasteur, Paris 79, 390.

Montagnes, D.J.S., 1996. Growth responses of planktonic ciliates in the genera Strobilidium and Strombidium. Marine Ecology Progress Series 130 (1-3), 241-254.

Mullin, M.M., Stewart, E.F., Fuglister, F.J., 1975. Ingestion by planktonic grazers as a function of food concentration. Limnology and Oceanography 20 (2), 259-262.

Murdoch, W.W., 1969. Switching in general predators: experiments on prey specificity and stability of prey populations. Ecological Monographs 39, 335-354.

Murdoch, W.W., 1973. The functional response of predators. Journal of Applied Ecology 10, 335-342.

Oaten, A., Murdoch, W.W., 1975a. Functional response and stability in predator-prey systems. American Naturalist 109, 289-298.

Oaten, A., Murdoch, W.W., 1975b. Switching functional response and stability in predator-prey systems. American Naturalist 109, 299-318.

Ohman, M.D., 1984. Omnivory by Euphausia pacifica: the role of copepod prey. Marine Ecology Progress Series 19, $125-131$.

Oreskes, N., Shrader-Frechette, K., Belitz, K., 1994. Verification, validation and confirmation of numerical models in the earth sciences. Science 263, 641-646.

Pace, M.L., Glasser, J.E., Pomeroy, L.R., 1984. A simulation analysis of continental shelf food webs. Marine Biology 82, 47-63.

Pahl-Wostl, C., 1997. Dynamic structure of a food web model: comparison with a food chain model. Ecological Modeling 100, 103-123.

Pitchford, J.W., Brindley, J., 1999. Iron limitation, grazing pressure and oceanic high nutrient-low Chlorophyll (HNLC) regions. Journal of Plankton Research 21 (3), 525-547.

Polis, G.A., Strong, D.R., 1996. Food-web complexity and community dynamics. The American Naturalist 147 (5), 813-846. 
Reeve, M.R., Walter, M.A., 1977. Observations on the existence of lower threshold and upper critical food concentration for the copepod Acartia tonsa Dana. Journal of Experimental Marine Biology and Ecology 29 (3), 211-221.

Rothschild, B.J., Osborn, T.R., 1988. Small-scale turbulence and plankton contact rates. Journal of Plankton Research $10,465-474$.

Sell, A.F., Van Keuren, D., Madin, L.P., 2001. Predation by omnivorous copepods on early developmental stages of Calanus finmarchicus and Pseudocalanus spp. Limnology and Oceanography 46 (4), 953-959.

Solomon, M.E., 1949. The natural control of animal populations. Journal of Animal Ecology 18, 1-35.

Stephens, D.W., Krebs, J.R., 1986. Foraging Theory. Princeton University Press, Princeton.

Stoecker, D.K., Cucci, T.L., Hulburt, E.M., Yentsch, C.M., 1986. Selective feeding by Balanion sp. (Ciliata: Balanionidae) on phytoplankton that best support its growth. Journal of Experimental Marine Biology and Ecology 95 (2), 113-130.

Strom, S.L., 1991. Growth and grazing rates of herbivorous dinoflagellate Gymnodinium sp. from the open subarctic Pacific Ocean. Marine Ecology Progress Series 78 (2), 103-113.
Strom, S.L., Loukos, H., 1998. Selective feeding by protozoa: model and experimental behaviors and their consequences for population stability. Journal of Plankton Research 20 (5), 831-846.

Strom, S.L., Miller, C.B., Frost, B.W., 2000. What sets lower limits to phytoplankton stocks in high-nitrate, low-Chlorophyll regions of the open ocean? Marine Ecology Progress Series 193, 19-31.

Tansky, M., 1978. Switching effect in a prey-predator system. Journal of Theoretical Biology 70, 263-271.

Van Gemerden, H., 1974. Coexistence of organisms competing for the same substrate: an example among the purple sulfur bacteria. Microbial Ecology 1, 104-119.

Veldkamp, H., Jannasch, H.W., 1972. Mixed culture studies with the chemostat. Journal of Applied Chemistry and Biotechnology 22, 105-123.

Verity, P.G., 1991. Measurement and simulation of prey uptake by marine planktonic ciliates fed plastidic and aplastidic nanoplankton. Limnology and Oceanography 36 (4), 729-750.

Wickham, S.A, 1995. Cyclops predation on ciliates: speciesspecific differences and functional response. Journal of Plankton Research 17 (8), 1633-1646. 